\title{
Pressure Stability in Fractional Step Finite Element Methods for Incompressible Flows
}

\author{
Ramon Codina \\ Universitat Politècnica de Catalunya, Jordi Girona 1-3, Edifici C1, 08034 Barcelona, Spain \\ E-mail: ramon.codina@upc.es
}

Received March 17, 2000; revised November 30, 2000

\begin{abstract}
The objective of this paper is to analyze the pressure stability of fractional step finite element methods for incompressible flows that use a pressure Poisson equation. For the classical first-order projection method, it is shown that there is a pressure control which depends on the time step size, and therefore there is a lower bound for this time step for stability reasons. The situation is much worse for a second-order scheme in which part of the pressure gradient is kept in the momentum equation. The pressure stability in this case is extremely weak. To overcome these shortcomings, a stabilized fractional step finite element method is also considered, and its stability is analyzed. Some simple numerical examples are presented to support the theoretical results. (c) 2001 Academic Press
\end{abstract}

\section{INTRODUCTION}

Fractional step methods for the incompressible Navier-Stokes equations have enjoyed widespread popularity since the original works of Chorin [1] and Temam [2]. The reason for this relies on the computational efficiency of these methods (see e.g., [3-6]), basically because of the uncoupling of the pressure from the velocity components. However, several issues related to these methods still deserve further analysis, and perhaps the most salient of these are the behavior of the computed pressure near boundaries and the stability of the pressure itself.

Referring to the pressure boundary conditions, it is well known that numerical boundary layers may appear. The reason for this can be explained by considering the pressure boundary condition associated to the splitting of the continuous problem, as proposed in $[1,2]$. Although relevant in some cases, this misbehavior does not affect the global pressure convergence [7], and can be shown to be less dramatic than expected in most situations [8]. In any case, any reference to the correct boundary conditions for the fractional step scheme can be skipped by considering the splitting at the purely algebraic level, once the 
space discretization has been performed. This is the approach advocated in $[9,10]$ and that we will follow here.

The study of the pressure stability for schemes that use a pressure Poisson equation is the main concern of this paper. Surprisingly, this stability is rarely made explicit. It is normally hidden by the convergence analysis, when it is required that the time step size be small enough. In general, analyses at the continuous space level are based on the stability of the continuous pressure $[11,12]$, whereas when the space is discretized it relies on the stability of this discretization, either the finite element method as in [13] or a very simple finite difference setting as in [14]. Other attempts to study the pressure stability are presented in [15], where results much weaker than those presented here are stated (only for the scheme of Section 3.2 and assuming the time step size sufficiently large)

The results to be presented in this paper refer to two types of fractional step schemes, namely, the classical first-order projection method and a second-order algorithm based on the Crank-Nicolson discretization for the viscous and convective terms and a secondorder pressure splitting, leaving the pressure gradient at a given time level in the first step and computing its increment in the second one (see [12, 16-19] for different ideas related to second-order schemes). First, the stability of these schemes in the context of a finite element space discretization is analyzed in Section 3 using matrix arguments
It is shown that a certain pressure stability can be expected, regardless of the particular
discrete velocity-pressure spaces chosen. In fact, sometimes fractional step schemes that use
the pressure Poisson equation are considered as "stabilized" [20]. However, this stability
can be useful for the first-order scheme, but it is certainly too weak for the second-
order one. In both cases, though, it is given by the time step size, and hence this size
is limited from below simply to stabilize the space discretization. It is worth noting that this dependence of the stabilization terms on the time step size also appears in some

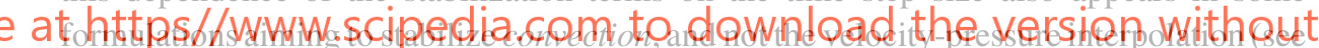

[21] for a version of the Characteristic-Galerkin method where this fact is clearly demonstrated).

To avoid the bond described, a pressure stabilized scheme is proposed in Section 4. Again, the stability analysis of both the first- and second-order fractional step schemes is undertaken, now using a variational setting rather than the previous matrix language. This stabilization is intended to mimic the stabilizing effect of the first-order projection method. The method was originally presented in [22] for the steady Stokes problem, extended to the nonlinear case in [23], and to the transient problem using a monolithic time discretization in [24]. In spite of the fact that the variational approach used in Section 4 supersedes the matrix analysis of Section 3, the latter is extremely useful to understand the stabilization mechanism introduced by the splitting and, more precisely, by approximation (14) in Section 2.

The final step is to extend the previous stabilization method to the case in which convection needs also to be stabilized. The resulting formulation, based on the ideas of [25], is presented in Section 5. The formulation is able to have control over the pressure gradient and the nonlinear convective term while yielding very accurate numerical results, much less overdiffusive than classical pressure stabilization methods or upwind techniques designed to deal with convection dominated flows.

Some numerical results are presented in Section 6. In spite of their simplicity, they clearly show that the theoretical predictions of the paper are encountered in practice. The summary of the most salient results and the conclusions are finally presented in Section 7. 
The pressure value computed here has been identified as the pressure evaluated at $t^{n+1}$, although this is irrelevant for the velocity approximation. The values of interest of $\theta$ are $\theta=1 / 2$, corresponding to the second-order Crank-Nicolson scheme (see [26] for a thorough analysis of this scheme) and $\theta=1$, which corresponds to the backward Euler method. If $\boldsymbol{f}$ is not continuous in time, $\left\langle\boldsymbol{f}^{n+\theta}, \boldsymbol{v}\right\rangle$ can be taken as the time average of $\langle\boldsymbol{f}, \boldsymbol{v}\rangle$ over the time step $\left[t^{n}, t^{n+1}\right]$.

2.1.3. Finite element discretizations. Let $\boldsymbol{V}_{h}$ be a finite element space to approximate $\boldsymbol{V}$, and $Q_{h}$ a finite element approximation to $Q$. Functions in $\boldsymbol{V}_{h}$ need to be continuous piecewise polynomials, whereas continuity is in principle not required for $Q_{h}$. However, we will consider only continuous pressure interpolations, for reasons explained below.

The finite element discretization of (3) and (4) reads

$\left(\delta_{t} \boldsymbol{u}_{h}^{n}, \boldsymbol{v}_{h}\right)+c\left(\boldsymbol{u}_{h}^{n+\theta}, \boldsymbol{u}_{h}^{n+\theta}, \boldsymbol{v}_{h}\right)+a\left(\boldsymbol{u}_{h}^{n+\theta}, \boldsymbol{v}_{h}\right)-b\left(p_{h}^{n+1}, \boldsymbol{v}_{h}\right)=\left\langle\boldsymbol{f}^{n+1}, \boldsymbol{v}_{h}\right\rangle \quad \forall \boldsymbol{v}_{h} \in \boldsymbol{V}_{h}$, $b\left(q_{h}, \boldsymbol{u}_{h}^{n+1}\right)=0 \quad \forall q_{h} \in Q_{h}$.

It is well known that for this discrete problem to be stable the velocity and pressure spaces need to satisfy the classical inf-sup condition (see e.g., [27]), which in particular precludes
the use of convenient equal velocity-pressure interpolations. However, it was early noted
that this condition is not required when fractional step methods using a pressure Poisson
equation are employed (see e.g.. [20,28-31]). The analysis and clarification of this situation
is precisely the objective of this paper.
Before presenting the fractional step schemes to be studied, let us introduce the matrix form of the problem. This is given by

e at https//www.scipedia.com to download the version without th

$$
\begin{gathered}
\mathrm{M} \delta_{t} \mathrm{U}^{n}+\mathrm{K}\left(\mathrm{U}^{n+\theta}\right) \mathrm{U}^{n+\theta}+\mathrm{GP}^{n+1}=\mathrm{F}^{n+\theta}, \\
\mathrm{DU}^{n+1}=0,
\end{gathered}
$$

where $U$ and $P$ are the arrays of nodal velocities and pressures, respectively. If we denote the node indexes with superscripts $a, b$, the space indexes with subscripts $i, j$, and the standard shape function of node $a$ by $N^{a}$, the components of the arrays involved in these equations are

$$
\begin{aligned}
\mathrm{M}_{i j}^{a b} & =\left(N^{a}, N^{b}\right) \delta_{i j} \quad\left(\delta_{i j} \text { is the Kronecker } \delta\right) \\
\mathrm{K}\left(\mathrm{U}^{n+\theta}\right)_{i j}^{a b} & =\left(N^{a}, \boldsymbol{u}_{h}^{n+\theta} \cdot \nabla N^{b}\right) \delta_{i j}+\frac{1}{2}\left(N^{a},\left(\nabla \cdot \boldsymbol{u}_{h}^{n+\theta}\right) N^{b}\right) \delta_{i j}+v\left(\nabla N^{a}, \nabla N^{b}\right) \delta_{i j} \\
\mathrm{G}_{i}^{a b} & =-\left(\partial_{i} N^{a}, N^{b}\right) \\
\mathrm{F}_{i}^{a} & =\left\langle N^{a}, f_{i}\right\rangle \\
\mathrm{D}_{j}^{a b} & =\left(N^{a}, \partial_{j} N^{b}\right) .
\end{aligned}
$$

It is understood that all the arrays are matrices (except $\mathrm{F}$, which is a vector) whose components are obtained by grouping together the left indexes in the previous expressions ( $a$ and possibly $i$ ) and the right indexes ( $b$ and possibly $j$ ). Note that $\mathrm{G}=-\mathrm{D}^{t}$. 


$$
\begin{gathered}
\delta t \mathrm{~L}\left(\mathrm{P}^{n+1}-\gamma \mathrm{P}^{n}\right)=\mathrm{D} \hat{U}^{n+1}, \\
\mathrm{M} \frac{1}{\delta t}\left(\mathrm{U}^{n+1}-\hat{\mathrm{U}}^{n+1}\right)+\mathrm{G}\left(\mathrm{P}^{n+1}-\gamma \mathrm{P}^{n}\right)=0 .
\end{gathered}
$$

Once arrived at this point, we may also write the discrete variational equations corresponding to this matrix problem, which are

$$
\begin{aligned}
& \frac{1}{\delta t}\left(\hat{\boldsymbol{u}}_{h}^{n+1}-\boldsymbol{u}_{h}^{n}, \boldsymbol{v}_{h}\right)+c\left(\hat{\boldsymbol{u}}_{h}^{n+\theta}, \hat{\boldsymbol{u}}_{h}^{n+\theta}, \boldsymbol{v}_{h}\right)+a\left(\hat{\boldsymbol{u}}_{h}^{n+\theta}, \boldsymbol{v}_{h}\right)-\gamma b\left(p_{h}^{n}, \boldsymbol{v}_{h}\right) \\
&=\left\langle\boldsymbol{f}^{n+1}, \boldsymbol{v}_{h}\right\rangle \quad \forall \boldsymbol{v}_{h} \in \boldsymbol{V}_{h}, \\
& \quad-\delta t\left(\nabla\left(p_{h}^{n+1}-\gamma p_{h}^{n}\right), \nabla q_{h}\right)=\left(q_{h}, \nabla \cdot \hat{\boldsymbol{u}}_{h}^{n+1}\right) \quad \forall q h \in Q_{h}, \\
& \frac{1}{\delta t}\left(u_{h}^{n+1}-\hat{u}_{h}^{n+1}, v_{h}\right)-b\left(p_{h}^{n+1}-\gamma p_{h}^{n}, v_{h}\right)=0 \quad \forall v_{h} \in V_{h},
\end{aligned}
$$

where $\hat{\boldsymbol{u}}_{h}$ is obviously the piecewise continuous function obtained by interpolating from the nodal values $\hat{U}$.

Formally, it is easy to see that the splitting error, introduced by approximation (10), is of order $O(\delta t)$ when $\gamma=0$ and of order $O\left(\delta t^{2}\right)$ when $\gamma=1$ (observe from (17) that
$O\left(\left\|U^{n+1}-U U^{n+1}\right\|\right)=\delta t O\left(\left\|P^{n+1}-\gamma P^{n}\right\|\right)$ in any nom $\left.\|\cdot\|\right)$. In order to haye the same
error resulting from the splitting and from the original time discretization, we will consider
two sets of parameters. The first is $\gamma=0, \theta=1$, which yields the classical first-order
projection scheme discretizing the continuous pressure Poisson equation, and the second
$\gamma=1, \theta=1 / 2$, which yields a (formally) second-order time accurate methed.

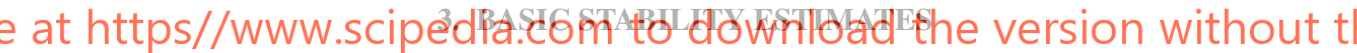

In this section we obtain stability estimates for the two schemes described above. We show that there is a certain pressure stability regardless of any compatibility requirement between the velocity and pressure approximations. In order to understand how is this possible, we show first how the fractional step scheme (15)-(17) can be viewed as a stabilized monolithic scheme. This equivalence allows us to predict that there will be a pressure stability inherent to the formulations.

\subsection{Equivalent Stabilized Monolithic Formulations}

In problem (15)-(17) we can eliminate either $\hat{U}$ or $U$, and think of the remaining variable as the approximation to the velocity. These two possibilities lead to two different stabilized formulations, as we show now.

Let us start by writing the problem only in terms of Û. Since

$$
\mathrm{U}^{n}=\hat{\mathrm{U}}^{n}-\delta t \mathrm{M}^{-1} \mathrm{G}\left(\mathrm{P}^{n}-\gamma \mathrm{P}^{n-1}\right),
$$

Eqs. (15)-(17) can be rewritten as

$$
\begin{gathered}
\mathrm{M} \frac{1}{\delta t}\left(\hat{\mathrm{U}}^{n+1}-\hat{\mathrm{U}}^{n}\right)+\mathrm{K}\left(\hat{\mathrm{U}}^{n+\theta}\right) \hat{\mathrm{U}}^{n+\theta}+\mathrm{G}\left[(1+\gamma) \mathrm{P}^{n}-\gamma \mathrm{P}^{n-1}\right]=\mathrm{F}^{n+1}, \\
\mathrm{D} \hat{\mathrm{U}}^{n+1}-\delta t \mathrm{~L}\left(\mathrm{P}^{n+1}-\gamma \mathrm{P}^{n}\right)=0 .
\end{gathered}
$$


and, on the other hand, if $\mathrm{M}_{i j}^{-1}$ are the components of $\mathrm{M}^{-1}$,

$$
\begin{aligned}
\mathrm{Q} \cdot \mathrm{DM}^{-1} \mathrm{GQ} & =-\sum_{i, j=1}^{n_{1}}\left(\int_{\Omega} \nabla q_{h} \cdot \boldsymbol{v}_{i} \mathrm{~d} \Omega\right) \mathrm{M}_{i j}^{-1}\left(\int_{\Omega} \nabla q_{h} \cdot \boldsymbol{v}_{j} \mathrm{~d} \Omega\right) \\
& =-\sum_{i, j=1}^{n_{1}} \sum_{k, l=1}^{n_{1}} \Pi_{1, k}\left(\int_{\Omega} \boldsymbol{v}_{k} \cdot \boldsymbol{v}_{i} \mathrm{~d} \Omega\right) \mathrm{M}_{i j}^{-1} \Pi_{1, l}\left(\int_{\Omega} \boldsymbol{v}_{l} \cdot \boldsymbol{v}_{j} \mathrm{~d} \Omega\right) \\
& =-\Pi_{1} \cdot \mathrm{M}_{1} .
\end{aligned}
$$

\section{Therefore,}

$$
\mathrm{Q} \cdot \mathrm{BQ}=\int_{\Omega} \pi_{23} \cdot \pi_{23} \mathrm{~d} \Omega \geq 0 .
$$

This proves that $B$ is positive semi-definite and explicitly shows that the components of $\nabla q_{h}$ controlled by this matrix are those orthogonal to the finite element space $V_{h}$, that is, $\pi_{23}$. This fact is used in [22] to prove that approximation (14) yields an error of the same order as the pressure interpolation error, and thus it does not deteriorate the accuracy of the finite elene boundaries.

At this point, it is convenient to introduce some additional not $\left\{\mathrm{X}^{n}\right\}_{n=0,1, \ldots, N}$ is a sequence of arrays of $N+\mathbb{1}$ terms and $\mathrm{A}$ a definite matrix, we define

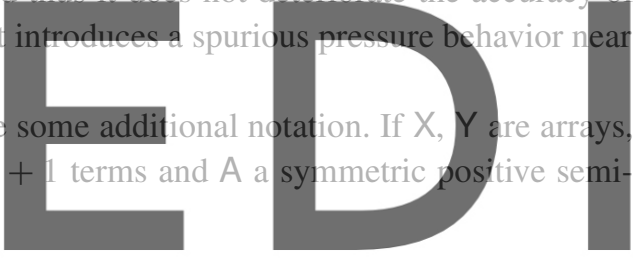

\section{at https//www.scipedia.com to download the version without th$$
\|Y\|_{-A}:=\sup _{x \neq 0} \frac{\|X\|_{A}}{4} \text { (here } A \text { is assumed to be positive definite), }
$$

$$
\|\mathrm{X}\|_{\mathrm{A}}:=(\mathrm{X} \cdot \mathrm{AX})^{1 / 2},
$$

$$
\begin{aligned}
& \left\{\mathrm{X}^{n}\right\} \in \ell^{\infty}(\mathrm{A}) \Leftrightarrow\left\|\mathrm{X}^{n}\right\|_{\mathrm{A}} \leq C<\infty \quad \forall n=0,1, \ldots, N, \\
& \left\{\mathrm{X}^{n}\right\} \in \ell^{p}(\mathrm{~A}) \Leftrightarrow \sum_{n=0}^{N} \delta t\left\|\mathrm{X}^{n}\right\|_{\mathrm{A}}^{p} \leq C<\infty, \quad 1 \leq p<\infty .
\end{aligned}
$$

Here and in the following, $C$ denotes a positive constant, not necessarily the same at different appearances.

A remark is needed when $A=K$. This matrix is not symmetric, but it has the contribution from the convective term, which is skew-symmetric, and the contribution from the viscous term, $\mathrm{K}_{\mathrm{visc}}$, which is symmetric and positive-definite. We will simply write $\mathrm{U} \cdot \mathrm{K}(\mathrm{U}) \mathrm{U}=$ $\mathrm{U} \cdot \mathrm{K}_{\mathrm{visc}} \mathrm{U} \equiv\|\mathrm{U}\|_{\mathrm{K}}^{2}$. We will make use also of $\mathrm{L}^{+}:=-\mathrm{L}$, which is the positive semi-definite matrix corresponding to the discretization of $-\Delta$.

These definitions will allow us to express our stability results in a compact manner. The basic assumption in all the cases will be that

$$
\sum_{n=0}^{N} \delta t\left\|\mathrm{~F}^{n}\right\|_{-\mathrm{K}}^{2} \leq C<\infty,
$$

which is the matrix version of the classical condition required for the problem to be well posed. 


\subsection{Stability of the First-Order Projection Method}

We will study now the stability properties of the first-order projection method, which corresponds to scheme (15)-(17) with $\gamma=0$ and $\delta=1$ :

$$
\begin{aligned}
\mathrm{M} \frac{1}{\delta t}\left(\hat{\mathrm{U}}^{n+1}-\mathrm{U}^{n}\right)+\mathrm{K}\left(\hat{\mathrm{U}}^{n+1}\right) \hat{\mathrm{U}}^{n+1} & =\mathrm{F}^{n+1}, \\
\delta t \mathrm{LP}^{n+1} & =\mathrm{D} \hat{\mathrm{U}}^{n+1}, \\
\mathrm{M} \frac{1}{\delta t}\left(\mathrm{U}^{n+1}-\hat{\mathrm{U}}^{n+1}\right)+\mathrm{GP}^{n+1} & =0 .
\end{aligned}
$$

The stability result for this scheme reads:

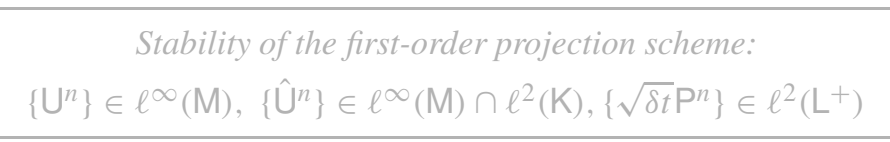

The stability estimate for the pressure shows that the pressure gradient multiplied by $\delta t$ is $\ell^{2}$ bounded. When $\delta t$ is of order $\mathcal{O}\left(h^{2}\right)$ this is optimal $[33,35,36]$.

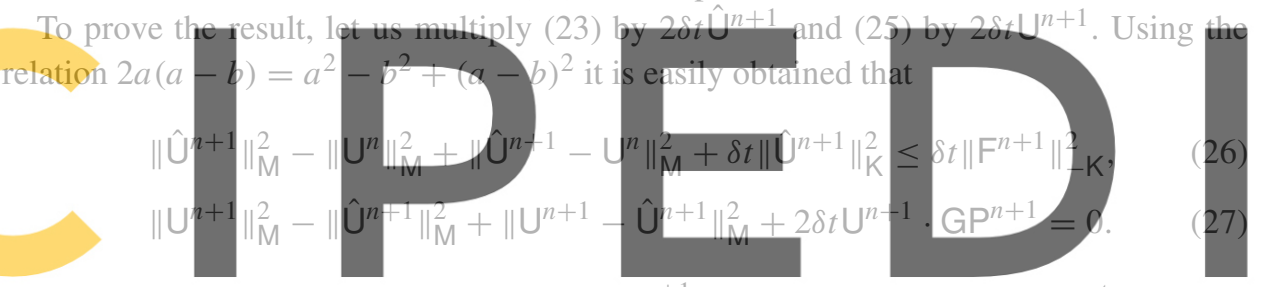

On the other hand, from (24) multiplied by $P^{n+1}$, using the fact that $G=-D^{t}$, and (20)

\section{at https 9 \%www wave}

$$
\begin{aligned}
\delta t \mathrm{P}^{n+1} \cdot \mathrm{LP}^{n+1} & =-\mathrm{U}^{n+1} \cdot \mathrm{GP}^{n+1}+\delta t \mathrm{P}^{n+1} \cdot \mathrm{DM}^{-1} \mathrm{GP}^{n+1}, \\
2 \delta t \mathrm{U}^{n+1} \cdot \mathrm{GP}^{n+1} & =2 \delta t^{2} \mathrm{P}^{n+1} \cdot \mathrm{BP}^{n+1}=2 \delta t\left\|\sqrt{\delta t} \mathrm{P}^{n+1}\right\|_{\mathrm{B}}^{2} .
\end{aligned}
$$

Using this in (27), adding up (26) and (27) and summing for $n$ (up to any $m \leq N$ ) it follows that

$$
\left\{\mathrm{U}^{n}\right\} \in \ell^{\infty}(\mathrm{M}), \quad\left\{\hat{\mathrm{U}}^{n}\right\} \in \ell^{2}(\mathrm{~K}), \quad\left\{\sqrt{\delta t} \mathrm{P}^{n}\right\} \in \ell^{2}(\mathrm{~B})
$$

On the other hand, (25) implies

$$
\left\|\mathrm{U}^{n+1}-\hat{\mathrm{U}}^{n+1}\right\|_{\mathrm{M}}^{2}=-\delta t^{2} \mathrm{P}^{n+1} \cdot \mathrm{DM}^{-1} \mathrm{GP}^{n+1},
$$

and from the definition of $B$ it is easy to see that

$$
\sum_{n=0}^{N} \delta t\left\|\sqrt{\delta t} \mathrm{P}^{n+1}\right\|_{\mathrm{L}^{+}}^{2}=\sum_{n=0}^{N} \delta t^{2} \mathrm{P}^{n+1} \cdot \mathrm{BP}^{n+1}+\sum_{n=0}^{N}\left\|\mathrm{U}^{n+1}-\hat{\mathrm{U}}^{n+1}\right\|_{\mathrm{M}}^{2} \cdot
$$

Both terms on the right-hand side are bounded. The first because we already know that $\left\{\sqrt{\delta t} \mathrm{P}^{n}\right\} \in \ell^{2}(\mathrm{~B})$, and the second because it is part of the contribution from (27) when it is added up with (26) and summed for $n$. Therefore,

$$
\left\{\sqrt{\delta t} \mathrm{P}^{n}\right\} \in \ell^{2}\left(\mathrm{~L}^{+}\right) .
$$


Likewise,

$$
\begin{aligned}
& \delta t \mathrm{U}^{n+1} \cdot \mathrm{G} \delta \mathrm{P}^{n}=-\delta t \delta \mathrm{P}^{n} \cdot \mathrm{D} \mathrm{U}^{n+1}=-\delta t \delta \mathrm{P}^{n} \cdot \mathrm{D}\left(\hat{\mathrm{U}}^{n+1}-\delta t \mathrm{M}^{-1} \mathrm{G} \delta \mathrm{P}^{n}\right) \\
& =-\delta t \delta \mathrm{P}^{n} \cdot\left(\delta t \mathrm{~L} \delta \mathrm{P}^{n}-\delta t \mathrm{DM}^{-1} \mathrm{G} \delta \mathrm{P}^{n}\right) \\
& =\delta t^{2} \delta \mathrm{P}^{n} \cdot \mathrm{B} \delta \mathrm{P}^{n}=\delta t\left\|\sqrt{\delta t} \delta \mathrm{P}^{n}\right\|_{\mathrm{B}}^{2} \text {. }
\end{aligned}
$$

Using (33)-(35) in (32), taking $\mathrm{P}^{0}=\mathrm{P}^{-1}=0$ and summing for $n$ we conclude that

$$
\left\{\mathrm{U}^{n}\right\} \in \ell^{\infty}(\mathrm{M}), \quad\left\{\hat{\mathrm{U}}^{n+1 / 2}\right\} \in \ell^{2}(\mathrm{~K}), \quad\left\{\delta t \mathrm{P}^{n}\right\} \in \ell^{\infty}\left(\mathrm{L}^{+}\right), \quad\left\{\sqrt{\delta t} \delta \mathrm{P}^{n}\right\} \in \ell^{2}\left(\mathrm{~L}^{+}\right) .
$$

It remains to show that $\left\{\hat{U}^{n}\right\} \in \ell^{\infty}(\mathrm{M})$. From (29) we have that

$$
\delta t \hat{\mathrm{U}}^{n+1} \cdot \mathrm{G} \delta \mathrm{P}^{n}=-\delta t \delta \mathrm{P}^{n} \mathrm{D} \hat{U}^{n+1}=\delta t^{2}\left\|\delta \mathrm{P}^{n}\right\|_{\mathrm{L}^{+}}^{2},
$$

which substituted in (31) yields, after making use of (35),

$$
\left\|\hat{\mathrm{U}}^{n+1}\right\|_{\mathrm{M}}^{2}=\left\|\mathrm{U}^{n+1}\right\|_{\mathrm{M}}^{2}+\delta t^{2}\left\|\delta \mathrm{P}^{n}\right\|_{\mathrm{L}^{+}}^{2}+\delta t^{2}\left\|\delta \mathrm{P}^{n}\right\|_{\mathrm{B}}^{2}
$$

The result follows noting that all the right-hand side terms are $\ell^{\infty}$ sequences.

\section{PRESSURE STABILIZED SCHEMES}

\subsection{Pressure Stabilization}

It has been shown in the previous section that the pressure stability of both the first- and second-order schemes depends on the time step size. It can be anticipated that if $\delta t$ is very small, stability problems may occur, especially for the second-order scheme. In order to avoid this, one can resort to a stabilized formulation, in the same spirit as for monolithic schemes.

Since the stability of the first order scheme is known to be adequate when $\delta t$ is taken close to the critical time step of the explicit scheme $(\theta=0$ in (5)), it seems natural to devise a stabilized formulation which inherits the stabilization effect of this first order scheme. This idea was first developed in [22] for the Stokes problem, and is briefly reproduced here for the transient Navier-Stokes equations and used in conjunction with a fractional step method.

Let $\tilde{D}, \tilde{M}$, and $\tilde{G}$ be the matrices with the same components as $D, M$, and $G$, respectively, but letting the velocity shape functions run over the boundary nodes also. Starting with the monolithic time discretization, the stabilized formulation we consider is

$$
\begin{gathered}
\mathrm{M} \delta_{t} \mathrm{U}^{n}+\mathrm{K}\left(\mathrm{U}^{n+\theta}\right) \mathrm{U}^{n+\theta}+\mathrm{GP}^{n+1}=\mathrm{F}^{n+\theta}, \\
\mathrm{DU}^{n+1}+\tau\left(\tilde{\mathrm{D}} \Pi^{n+\beta}-\mathrm{LP}^{n+1}\right)=0, \\
\tilde{\mathrm{M}} \Pi^{n+1}=\tilde{\mathrm{G}} \mathrm{P}^{n+1},
\end{gathered}
$$

where $\Pi$ is an auxiliary variable, which may be treated either implicitly if $\beta=1$ or explicitly if $\beta=0$, and $\tau$ is a stabilization parameter which depends on the local element sizes. For the sake of simplicity in the exposition, we will take the finite element meshes quasi-uniform, 
and thus $\tau$ will depend only on the diameter of the finite element partition $h$. The stability and convergence analysis dictates that it must behave as

$$
\tau \leq C \frac{h^{2}}{v}
$$

for viscous dominated cases (an appropriate choice for the constant is $C=1 / 4$ when linear elements are used). In general, it can be taken close to the critical time step of the explicit time integration of (5) and (6) (see [22, 24] for further discussion). Nevertheless, the exact value of $\tau$ is irrelevant for our discussion.

To see that the modified monolithic problem will inherit the type of stability of the firstorder projection method, note that if $\beta=1$ and $\Pi$ is eliminated, the modified continuity equation is

$$
\mathrm{DU}^{n+1}+\tau \tilde{\mathrm{B}} \mathrm{P}^{n+1}=0, \quad \tilde{\mathrm{B}}:=\tilde{\mathrm{D}} \tilde{\mathrm{M}}^{-1} \tilde{\mathrm{G}}-\mathrm{L}
$$

which is similar to (21) with $\gamma=0$.

The reason for having modified matrices $D, M$ and $G$ to $\tilde{D}, \tilde{M}$ and $\tilde{G}$, respectively, is that in this way the modified monolithic problem does not suffer from spurious pressure boundary layers, as it happens for the first-order projection scheme (see [22] for the analysis of this point).

For the monolithic scheme we may use the same approximations (10) and (14) as for the nonstabilized (Galerkin) formulation to arrive to the split problem:

$$
\begin{gathered}
\mathrm{M} \frac{1}{\delta t}\left(\hat{\mathrm{U}}^{n+1}-\mathrm{U}^{n}\right)+\mathrm{K}\left(\hat{\mathrm{U}}^{n+\theta}\right) \hat{\mathrm{U}}^{n+\theta}+\gamma \mathrm{GP}^{n}=\mathrm{F}^{n+\theta}, \\
\delta t \mathrm{~L}\left(\mathrm{P}^{n+1}-\gamma \mathrm{P}^{n}\right)+\tau\left(\mathrm{LP}^{n+1}-\tilde{\mathrm{D}} \Pi^{n+\beta}\right)=\mathrm{D} \hat{U}^{n+1}, \\
\mathrm{M} \frac{1}{\delta t}\left(\mathrm{U}^{n+1}-\hat{\mathrm{U}}^{n+1}\right)+\mathrm{G}\left(\mathrm{P}^{n+1}-\gamma \mathrm{P}^{n}\right)=0, \\
\tilde{\mathrm{M}} \Pi^{n+1}=\tilde{\mathrm{G}} \mathrm{P}^{n+1} .
\end{gathered}
$$

The variational statement corresponding to this matrix problem, which we will use in this section, consists of finding $\hat{\boldsymbol{u}}_{h}^{n+1} \in \boldsymbol{V}_{h}, p_{h}^{n+1} \in Q_{h}, \boldsymbol{u}_{h}^{n+1} \in \boldsymbol{V}_{h}$, and $\pi_{h}^{n+1} \in \tilde{\boldsymbol{V}}_{h}$ such that

$$
\begin{gathered}
\frac{1}{\delta t}\left(\hat{\boldsymbol{u}}_{h}^{n+1}-\boldsymbol{u}_{h}^{n}, \boldsymbol{v}_{h}\right)+c\left(\hat{\boldsymbol{u}}_{h}^{n+\theta}, \hat{\boldsymbol{u}}_{h}^{n+\theta}, \boldsymbol{v}_{h}\right)+a\left(\hat{\boldsymbol{u}}_{h}^{n+\theta}, \boldsymbol{v}_{h}\right)-\gamma b\left(p_{h}^{n}, \boldsymbol{v}_{h}\right) \\
=\left\langle\boldsymbol{f}^{n+\theta}, \boldsymbol{v}_{h}\right\rangle \quad \forall \boldsymbol{v}_{h} \in \boldsymbol{V}_{h}, \\
-\delta t\left(\nabla\left(p_{h}^{n+1}-\gamma p_{h}^{n}\right), \nabla q_{h}\right)-\tau\left(\nabla p_{h}^{n+1}-\pi_{h}^{n+\beta}, \nabla q_{h}\right) \\
=\left(q_{h}, \nabla \cdot \hat{\boldsymbol{u}}_{h}^{n+1}\right) \quad \forall q_{h} \in Q_{h}, \\
\frac{1}{\delta t}\left(\boldsymbol{u}_{h}^{n+1}-\hat{\boldsymbol{u}}_{h}^{n+1}, \boldsymbol{v}_{h}\right)-b\left(p_{h}^{n+1}-\gamma p_{h}^{n}, \boldsymbol{v}_{h}\right)=0 \quad \forall \boldsymbol{v}_{h} \in \boldsymbol{V}_{h}, \\
\left(\boldsymbol{\pi}_{h}^{n+1}, \boldsymbol{\eta}_{h}\right)=\left(\nabla p_{h}^{n+1}, \boldsymbol{\eta}_{h}\right) \quad \forall \boldsymbol{\eta}_{h} \in \tilde{\boldsymbol{V}}_{h},
\end{gathered}
$$

where $\tilde{\boldsymbol{V}}_{h}$ is the space $\boldsymbol{V}_{h}$ enlarged with the continuous vector functions associated to the boundary nodes. The meaning of the new auxiliary variable $\pi_{h}$ is clearly observed from (39): It is the projection of the pressure gradient $\nabla p_{h}$ onto $\tilde{\boldsymbol{V}}_{h}$. 
In the following analysis, we will need several projections of $\nabla p_{h}$, all of them with respect to the $L^{2}$-inner product. These projections are

$$
\begin{aligned}
& \boldsymbol{\pi}_{1} \text { : projection onto } \boldsymbol{V}_{h}, \\
& \boldsymbol{\pi}_{2} \text { : projection onto } \boldsymbol{V}_{h}^{\perp} \cap \tilde{\boldsymbol{V}}_{h}, \\
& \boldsymbol{\pi}_{3} \text { : projection onto } \tilde{\boldsymbol{V}}_{h}^{\perp} .
\end{aligned}
$$

Likewise, we will denote $\pi_{i j}:=\pi_{i}+\pi_{j}$. Observe in particular that $\pi_{12} \equiv \pi_{h}$, and that, using these projections, (38) implies

$$
\boldsymbol{u}_{h}^{n+1}=\hat{\boldsymbol{u}}_{h}^{n+1}-\delta t\left(\boldsymbol{\pi}_{1}^{n+1}-\gamma \boldsymbol{\pi}_{1}^{n}\right) .
$$

We will see from the following analysis that the terms multiplied by $\tau$ provide control on $\pi_{3}$, that is, the component of the pressure gradient orthogonal to the space of continuous vector fields. Control on $\pi_{1}$ can be directly obtained from the momentum equation, as we will see, whereas control over $\pi_{2}$ follows from the condition

$$
\left\|\nabla p_{h}\right\| \leq C\left(\left\|\pi_{1}\right\|+\left\|\pi_{3}\right\|\right)
$$

which is assumed to hold for any $p_{h} \in Q_{h}$. This condition is studied in detail in [22]. In particular, it is shown to hold when equal interpolation is used for the velocity components and the pressure, the situation in which we are interested.

Similarly to Section 3, the stability estimates we will obtain here will be expressed using the following notation. Let $\left\{f^{n}\right\}$ be a sequence of generic functions (scalar or vectorial). We define

$$
\begin{aligned}
& \left\{f^{n}\right\} \in \ell^{\infty}\left(L^{2}\right) \Leftrightarrow\left\|f^{n}\right\| \leq C<\infty \quad \forall n=0,1, \ldots, N, \\
& \left\{f^{n}\right\} \in \ell^{p}\left(L^{2}\right) \Leftrightarrow \sum_{n=0}^{N} \delta t\left\|f^{n}\right\|^{p} \leq C<\infty, \quad 1 \leq p<\infty, \\
& \left\{f^{n}\right\} \in \ell^{2}\left(H^{1}\right) \Leftrightarrow \sum_{n=0}^{N} \delta t\left\|f^{n}\right\|_{1}^{2} \leq C<\infty,
\end{aligned}
$$

where $\|\cdot\|$ is the standard $L^{2}$ norm and $\|\cdot\|_{1}$ is the $H^{1}$ norm, that is, the sum of the $L^{2}$ norm of a function and the $L^{2}$ norm of its gradient. We recall that for functions $f$ vanishing on $\partial \Omega,\|f\|_{1}$ is equivalent to $\|\nabla f\|$. Likewise, we will use the fact that $\ell^{2}\left(L^{2}\right) \subset \ell^{1}\left(L^{2}\right)$, valid for finite time intervals of analysis.

Finally, let us state the condition analogous to (22) in the variational setting that we use now, which is

$$
\sum_{n=0}^{N} \delta t\left\|\boldsymbol{f}^{n}\right\|_{-1}^{2} \leq C<\infty
$$

where $\|\cdot\|_{-1}$ is the dual norm of $\|\cdot\|_{1}$. 


\subsection{Stability of the Stabilized First-Order Scheme}

The first-order stabilized scheme is obtained taking $\gamma=0$ and $\theta=1$ in (36)-(39), which yields

$$
\begin{gathered}
\frac{1}{\delta t}\left(\hat{\boldsymbol{u}}_{h}^{n+1}-\boldsymbol{u}_{h}^{n}, \boldsymbol{v}_{h}\right)+c\left(\hat{\boldsymbol{u}}_{h}^{n+1}, \hat{\boldsymbol{u}}_{h}^{n+1}, \boldsymbol{v}_{h}\right)+a\left(\hat{\boldsymbol{u}}_{h}^{n+1}, \boldsymbol{v}_{h}\right)=\left\langle\boldsymbol{f}^{n+1}, \boldsymbol{v}_{h}\right\rangle \quad \forall \boldsymbol{v}_{h} \in \boldsymbol{V}_{h}, \\
-\delta t\left(\nabla p_{h}^{n+1}, \nabla q_{h}\right)-\tau\left(\nabla p_{h}^{n+1}-\boldsymbol{\pi}_{h}^{n+\beta}, \nabla q_{h}\right)=\left(q_{h}, \nabla \cdot \hat{\boldsymbol{u}}_{h}^{n+1}\right) \quad \forall q_{h} \in Q_{h}, \\
\frac{1}{\delta t}\left(\boldsymbol{u}_{h}^{n+1}-\hat{\boldsymbol{u}}_{h}^{n+1}, \boldsymbol{v}_{h}\right)-b\left(p_{h}^{n+1}, \boldsymbol{v}_{h}\right)=0 \quad \forall \boldsymbol{v}_{h} \in \boldsymbol{V}_{h} .
\end{gathered}
$$

The stability result for this formulation is:

$$
\begin{aligned}
& \text { Stability of the stabilized first-order scheme: } \\
& \left\{u_{h}^{n}\right\} \in \ell^{\infty}\left(L^{2}\right), \quad\left\{\hat{\boldsymbol{u}}_{h}^{n}\right\} \in \ell^{\infty}\left(L^{2}\right) \cap \ell^{2}\left(H^{1}\right), \\
& \left\{\sqrt{\delta t} \nabla p_{h}^{n}\right\} \in \ell^{2}\left(L^{2}\right), \quad\left\{\sqrt{\tau} \nabla p_{h}^{n}\right\} \in \ell^{1}\left(L^{2}\right)
\end{aligned}
$$

From this we see that, on top of the stability estimate of the scheme with $\tau=0$, now we have an additional one which is optimal if $\tau=\mathcal{O}\left(h^{2}\right)$.

Let us proceed to prove these stability results. Taking $\boldsymbol{v}_{h}=2 \delta t \hat{\boldsymbol{u}}_{h}^{n+1}$ in (42) and $\boldsymbol{v}_{h}=$ $2 \delta t \boldsymbol{u}_{h}^{n+1}$ in (44) it is found that

$$
\begin{aligned}
& \left\|\hat{\boldsymbol{u}}_{h}^{n+1}\right\|^{2}-\left\|\boldsymbol{u}_{h}^{n}\right\|^{2}+\left\|\hat{\boldsymbol{u}}_{h}^{n+1}-\boldsymbol{u}_{h}^{n}\right\|^{2}+\delta t v\left\|\nabla \hat{\boldsymbol{u}}_{h}^{n+1}\right\|^{2} \leq C \delta t\left\|\boldsymbol{f}^{n+1}\right\|_{-1}^{2}, \\
& \left\|\boldsymbol{u}_{h}^{n+1}\right\|^{2}-\left\|\hat{\boldsymbol{u}}_{h}^{n+1}\right\|^{2}+\left\|\boldsymbol{u}_{h}^{n+1}-\hat{\boldsymbol{u}}_{h}^{n+1}\right\|^{2}+\delta t\left(p_{h}^{n+1}, \nabla \cdot \boldsymbol{u}_{h}^{n+1}\right)=0 .
\end{aligned}
$$

On the other hand, from (43) and using (40) (with $\gamma=0$ ) we have that

$$
\begin{aligned}
-\left(p_{h}^{n+1}, \nabla \cdot \boldsymbol{u}_{h}^{n+1}\right) & =\left(\nabla p_{h}^{n+1}, \hat{\boldsymbol{u}}_{h}^{n+1}\right)-\delta t\left(\nabla p_{h}^{n+1}, \boldsymbol{\pi}_{1}^{n+1}\right) \\
& =\delta t\left(\nabla p_{h}^{n+1}, \nabla p_{h}^{n+1}\right)+\tau\left(\nabla p_{h}^{n+1}, \nabla p_{h}^{n+1}-\boldsymbol{\pi}_{h}^{n+\beta}\right)-\delta t\left(\boldsymbol{\pi}_{1}^{n+1}, \boldsymbol{\pi}_{1}^{n+1}\right) \\
& =\delta t\left\|\boldsymbol{\pi}_{23}^{n+1}\right\|^{2}+\tau\left(\nabla p_{h}^{n+1}, \nabla p_{h}^{n+1}-\boldsymbol{\pi}_{h}^{n+\beta}\right) .
\end{aligned}
$$

Calling $\bar{\beta}:=1-\beta$, the second term in the right-hand side can be written as

$$
\begin{aligned}
\left(\nabla p_{h}^{n+1}, \nabla p_{h}^{n+1}-\pi_{h}^{n+\beta}\right) & =\left(\nabla p_{h}^{n+1}, \boldsymbol{\pi}_{3}^{n+1}\right)+\bar{\beta}\left(\nabla p_{h}^{n+1}, \boldsymbol{\pi}_{h}^{n+1}-\boldsymbol{\pi}_{h}^{n}\right) \\
& =\left\|\boldsymbol{\pi}_{3}^{n+1}\right\|^{2}+\frac{\bar{\beta}}{2}\left(\left\|\boldsymbol{\pi}_{h}^{n+1}\right\|^{2}-\left\|\boldsymbol{\pi}_{h}^{n}\right\|^{2}+\left\|\boldsymbol{\pi}_{h}^{n+1}-\boldsymbol{\pi}_{h}^{n}\right\|^{2}\right) .
\end{aligned}
$$

Using this in (47), the result in (46), adding it with (45), and summing for $n$ it is found that

$$
\begin{gathered}
\left\{\boldsymbol{u}_{h}^{n}\right\} \in \ell^{\infty}\left(L^{2}\right), \quad\left\{\hat{\boldsymbol{u}}_{h}^{n}\right\} \in \ell^{2}\left(H^{1}\right), \\
\left\{\sqrt{\delta t} \boldsymbol{\pi}_{23}^{n}\right\} \in \ell^{2}\left(L^{2}\right) . \quad\left\{\sqrt{\tau} \boldsymbol{\pi}_{3}^{n}\right\} \in \ell^{2}\left(L^{2}\right) .
\end{gathered}
$$

On the other hand, from (40) again,

$$
\left\|\boldsymbol{u}_{h}^{n+1}-\hat{\boldsymbol{u}}_{h}^{n+1}\right\|^{2}=\delta t^{2}\left\|\boldsymbol{\pi}_{1}^{n+1}\right\|^{2},
$$


and since the sum for $n$ of the left-hand side is bounded (it appears when (45) and (46) are added) we have that $\left\{\sqrt{\delta t} \pi_{1}^{n}\right\} \in \ell^{2}\left(L^{2}\right)$, which together with the fact that $\left\{\sqrt{\delta t} \pi_{23}^{n}\right\} \in$ $\ell^{2}\left(L^{2}\right)$, implies that $\left\{\sqrt{\delta t} \nabla p_{h}^{n}\right\} \in \ell^{2}\left(L^{2}\right)$.

Stability of $\left\{\sqrt{\tau} \pi_{1}^{n}\right\}$ is more delicate. It is provided by the momentum equation actually solved by the fractional scheme, which after adding (42) and (44) is found to be

$$
\frac{1}{\delta t}\left(\boldsymbol{u}_{h}^{n+1}-\boldsymbol{u}_{h}^{n}, \boldsymbol{v}_{h}\right)+c\left(\hat{\boldsymbol{u}}_{h}^{n+1}, \hat{\boldsymbol{u}}_{h}^{n+1}, \boldsymbol{v}_{h}\right)+a\left(\hat{\boldsymbol{u}}_{h}^{n+1}, \boldsymbol{v}_{h}\right)+\left(\nabla p_{h}^{n+1}, \boldsymbol{v}_{h}\right)=\left\langle\boldsymbol{f}^{n+1}, \boldsymbol{v}_{h}\right\rangle .
$$

Taking $\boldsymbol{v}_{h}=\pi_{1}^{n+1}$ and using the standard inverse estimate (see e.g., [39])

$$
\left\|\pi_{1}^{n+1}\right\|_{1} \leq \frac{C_{\mathrm{inv}}}{h}\left\|\pi_{1}^{n+1}\right\|
$$

valid for quasi-uniform finite element partitions, we have

$$
\begin{aligned}
\left\|\boldsymbol{\pi}_{1}^{n+1}\right\|^{2} \leq & \left\|\boldsymbol{f}^{n+1}\right\|_{-1} \frac{C_{\mathrm{inv}}}{h}\left\|\boldsymbol{\pi}_{h}^{n+1}\right\|+\frac{1}{\delta t}\left\|\boldsymbol{u}_{h}^{n+1}-\boldsymbol{u}_{h}^{n}\right\|\left\|\boldsymbol{\pi}_{1}^{n+1}\right\| \\
& +\left(N_{a}\left\|\hat{\boldsymbol{u}}_{h}^{n+1}\right\|_{1}+N_{c}\left\|\hat{\boldsymbol{u}}_{h}^{n+1}\right\|_{1}^{2}\right) \frac{C_{\mathrm{inv}}}{h}\left\|\pi_{1}^{n+1}\right\|,
\end{aligned}
$$

where $N_{a}$ and $N_{c}$ are the norms of the forms $a$ and $c$, respectively, and we have used the continuity of $a$ and $c$, that is, $a(\boldsymbol{u}, \boldsymbol{v}) \leq N_{a}\|\boldsymbol{u}\|_{1}\|\boldsymbol{v}\|_{1}, c(\boldsymbol{u}, \boldsymbol{v}, \boldsymbol{w}) \leq N_{c}\|\boldsymbol{u}\|_{1}\|\boldsymbol{v}\|_{1}\|\boldsymbol{w}\|_{1}$. Dividing by $\left\|\pi_{1}^{n+1}\right\|$, summing for $n$, noting that $\sqrt{\tau} \leq C h$ and using the previous stability bounds, it is found that $\left\{\sqrt{\tau} \pi_{1}^{n}\right\} \in \ell^{2}\left(L^{2}\right)$. This, together with the fact that $\left\{\sqrt{\tau} \pi_{3}^{n}\right\} \in$ $\ell^{2}\left(L^{2}\right)$ and assumption (41), allows us to conclude that $\left\{\sqrt{\tau} \nabla p_{h}^{n}\right\} \in \ell^{2}\left(L^{2}\right)$. Observe that the reason why this result can not be improved to $\left\{\sqrt{\tau} \nabla p_{h}^{n}\right\} \in \ell^{2}\left(L^{2}\right)$ is the presence of the term $N_{c}\left\|\hat{\boldsymbol{u}}_{h}^{n+1}\right\|_{1}^{2}$ in (49) (see [24] for a discussion about the possibility to improve this result for the monolithic scheme).

Finally, the fact that $\left\{\hat{\boldsymbol{u}}_{h}^{n}\right\} \in \ell^{\infty}\left(L^{2}\right)$ follows adding (45) and (46) evaluated at $n$ instead of $n+1$ and summing for $n$.

\subsection{Stability of the Stabilized Second-Order Scheme}

To conclude this section, let us study the stabilized second-order method, which is obtained selecting $\gamma=1$ and $\theta=1 / 2$ in (36)-(39):

$$
\begin{aligned}
& \frac{1}{\delta t}\left(\hat{\boldsymbol{u}}_{h}^{n+1}-\boldsymbol{u}_{h}^{n}, \boldsymbol{v}_{h}\right)+c\left(\hat{\boldsymbol{u}}_{h}^{n+1 / 2}, \hat{\boldsymbol{u}}_{h}^{n+1 / 2}, \boldsymbol{v}_{h}\right)+a\left(\hat{\boldsymbol{u}}_{h}^{n+1 / 2}, \boldsymbol{v}_{h}\right)-b\left(p_{h}^{n}, \boldsymbol{v}_{h}\right) \\
& \quad=\left\langle\boldsymbol{f}^{n+1 / 2}, \boldsymbol{v}_{h}\right\rangle \quad \forall \boldsymbol{v}_{h} \in \boldsymbol{V}_{h}, \\
& -\delta t\left(\nabla\left(p_{h}^{n+1}-p_{h}^{n}\right), \nabla q_{h}\right)-\tau\left(\nabla p_{h}^{n+1}-\boldsymbol{\pi}_{h}^{n+\beta}, \nabla q_{h}\right) \\
& \quad=\left(q_{h}, \nabla \cdot \hat{\boldsymbol{u}}_{h}^{n+1}\right) \quad \forall q_{h} \in Q_{h}, \\
& \frac{1}{\delta t}\left(\boldsymbol{u}_{h}^{n+1}-\hat{\boldsymbol{u}}_{h}^{n+1}, \boldsymbol{v}_{h}\right)-b\left(p_{h}^{n+1}-p_{h}^{n}, \boldsymbol{v}_{h}\right)=0 \quad \forall \boldsymbol{v}_{h} \in \boldsymbol{V}_{h} .
\end{aligned}
$$


Contrary to what happened for the first-order scheme, now we need to require that $\tau \leq C \delta t$. The stability we are able to prove in this case is:

Stability of the stabilized second-order scheme:
$\left\{\boldsymbol{u}_{h}^{n}\right\} \in \ell^{\infty}\left(L^{2}\right), \quad\left\{\hat{\boldsymbol{u}}_{h}^{n}\right\} \in \ell^{\infty}\left(L^{2}\right), \quad\left\{\hat{\boldsymbol{u}}_{h}^{n+1 / 2}\right\} \in \ell^{2}\left(H^{1}\right)$,
$\left\{\delta t \nabla p_{h}^{n}\right\} \in \ell^{\infty}\left(L^{2}\right), \quad\left\{\sqrt{\delta t} \nabla \delta p_{h}^{n}\right\} \in \ell^{2}\left(L^{2}\right), \quad\left\{\sqrt{\tau} \nabla p_{h}^{n}\right\} \in \ell^{1}\left(L^{2}\right)$

The useful pressure stability estimate in this case is the last one, which again is optimal when $\tau=\mathcal{O}\left(h^{2}\right)$.

The proof-strategy is similar to the previous cases. Taking $\boldsymbol{v}_{h}=2 \delta t \hat{\boldsymbol{u}}_{h}^{n+1 / 2}$ in (50) and $\boldsymbol{v}_{h}=\delta t\left(\boldsymbol{u}_{h}^{n+1}+\hat{\boldsymbol{u}}_{h}^{n+1}\right)$ in (52) it is found that

$$
\begin{aligned}
& \left\|\hat{\boldsymbol{u}}_{h}^{n+1}\right\|-\left\|\boldsymbol{u}_{h}^{n}\right\|+\delta t v\left\|\nabla \hat{\boldsymbol{u}}_{h}^{n+1 / 2}\right\|^{2}-\delta t\left(p_{h}^{n}, \nabla \cdot \hat{\boldsymbol{u}}_{h}^{n+1}\right)-\delta t\left(p_{h}^{n}, \nabla \cdot \boldsymbol{u}_{h}^{n}\right) \\
& \quad \leq C \delta t\left\|\boldsymbol{f}^{n+1 / 2}\right\|_{-1}^{2}, \\
& \quad\left\|\boldsymbol{u}_{h}^{n+1}\right\|^{2}-\left\|\hat{\boldsymbol{u}}_{h}^{n+1}\right\|^{2}-\delta t\left(p_{h}^{n+1}-p_{h}^{n}, \nabla \cdot \boldsymbol{u}_{h}^{n+1}\right)-\delta t\left(p_{h}^{n+1}-p_{h}^{n}, \nabla \cdot \hat{\boldsymbol{u}}_{h}^{n+1}\right)=0 .
\end{aligned}
$$

Adding these two expressions we get

$$
\begin{aligned}
& \left\|\boldsymbol{u}_{h}^{n+1}\right\|^{2}-\left\|\boldsymbol{u}_{h}^{n}\right\|^{2}+\delta t v\left\|\nabla \hat{\boldsymbol{u}}_{h}^{n+1 / 2}\right\|^{2}-\delta t\left(p_{h}^{n}, \nabla \cdot \boldsymbol{u}_{h}^{n}\right)-\delta t\left(p_{h}^{n+1}-p_{h}^{n}, \nabla \cdot \boldsymbol{u}_{h}^{n+1}\right) \\
& \quad-\delta t\left(p_{h}^{n+1}, \nabla \cdot \hat{\boldsymbol{u}}_{h}^{n+1}\right) \leq C \delta t\left\|\boldsymbol{f}^{n+1 / 2}\right\|_{-1}^{2} .
\end{aligned}
$$

We need to deal with the different terms involving the pressure in the left-hand side of this inequality. Using (51) and (52), as well as (40), the various terms can be written as

$$
\begin{aligned}
-\left(p_{h}^{n}, \nabla \cdot \boldsymbol{u}_{h}^{n}\right)= & -\left(p_{h}^{n}, \nabla \cdot \hat{u}_{h}^{n}\right)+\delta t\left(p_{h}^{n}, \nabla \cdot\left(\boldsymbol{\pi}_{1}^{n}-\boldsymbol{\pi}_{1}^{n-1}\right)\right) \\
= & \delta t\left(\nabla \delta p_{h}^{n-1}, \nabla p_{h}^{n}\right)+\tau\left(\nabla p_{h}^{n}-\boldsymbol{\pi}_{h}^{n+\beta-1}, \nabla p_{h}^{n}\right) \\
& -\delta t\left(\nabla p_{h}^{n}, \boldsymbol{\pi}_{1}^{n}-\boldsymbol{\pi}_{1}^{n-1}\right) \\
= & \delta t\left(\boldsymbol{\pi}_{23}^{n}-\boldsymbol{\pi}_{23}^{n-1}, \nabla p_{h}^{n}\right)+\tau\left(\nabla p_{h}^{n}-\boldsymbol{\pi}_{h}^{n+\beta-1}, \nabla p_{h}^{n}\right) \\
= & \frac{1}{2} \delta t\left(\left\|\boldsymbol{\pi}_{23}^{n}\right\|^{2}-\left\|\boldsymbol{\pi}_{23}^{n-1}\right\|^{2}+\left\|\boldsymbol{\pi}_{23}^{n}-\boldsymbol{\pi}_{23}^{n-1}\right\|^{2}\right) \\
& +\tau\left(\nabla p_{h}^{n}-\boldsymbol{\pi}_{h}^{n+\beta-1}, \nabla p_{h}^{n}\right) \\
-\left(\delta p_{h}^{n}, \nabla \cdot \boldsymbol{u}_{h}^{n+1}\right)= & -\left(\delta p_{h}^{n}, \nabla \cdot \hat{\boldsymbol{u}}_{h}^{n+1}\right)+\delta t\left(\delta p_{h}^{n}, \nabla \cdot\left(\boldsymbol{\pi}_{1}^{n+1}-\boldsymbol{\pi}_{1}^{n}\right)\right) \\
= & \delta t\left(\nabla \delta p_{h}^{n}, \nabla \delta p_{h}^{n}\right)+\tau\left(\nabla p_{h}^{n+1}-\boldsymbol{\pi}_{h}^{n+\beta}, \nabla \delta p_{h}^{n}\right) \\
& -\delta t\left(\nabla \delta p_{h}^{n}, \boldsymbol{\pi}_{1}^{n+1}-\boldsymbol{\pi}_{1}^{n}\right) \\
= & \delta t\left\|\boldsymbol{\pi}_{23}^{n}-\boldsymbol{\pi}_{23}^{n}\right\|^{2}+\tau\left(\nabla p_{h}^{n+1}-\boldsymbol{\pi}_{h}^{n+\beta}, \nabla \delta p_{h}^{n}\right) \\
= & \delta t\left(\nabla \delta p_{h}^{n}, \nabla p_{h}^{n+1}\right)+\tau\left(\nabla p_{h}^{n+1}-\pi_{h}^{n+\beta}, \nabla p_{h}^{n+1}\right) \\
& \delta t \\
-\left(p_{h}^{n+1}, \nabla \cdot \hat{\boldsymbol{u}}_{h}^{n+1}\right) & \frac{2}{2}\left(\nabla p_{h}^{n+1}\left\|^{2}-\right\| \nabla p_{h}^{n}\left\|^{2}+\right\| \nabla p_{h}^{n+1}-\nabla p_{h}^{n} \|^{2}\right) \\
& +\tau\left(\nabla p_{h}^{n+1}-\boldsymbol{\pi}_{h}^{n+\beta}, \nabla p_{h}^{n+1}\right) .
\end{aligned}
$$


The terms multiplied by $\tau$ in these expressions can be written as

$$
\begin{aligned}
\left(\nabla p_{h}^{n+1}-\pi_{h}^{n+\beta}, \nabla p_{h}^{n+1}\right) & =\left(\pi_{3}^{n+1}, \nabla p_{h}^{n+1}\right)+\bar{\beta}\left(\boldsymbol{\pi}_{h}^{n+1}-\boldsymbol{\pi}_{h}^{n}, \nabla p_{h}^{n+1}\right) \\
& =\left\|\boldsymbol{\pi}_{3}^{n+1}\right\|^{2}+\frac{\bar{\beta}}{2}\left(\left\|\boldsymbol{\pi}_{h}^{n+1}\right\|^{2}-\left\|\boldsymbol{\pi}_{h}^{n}\right\|^{2}+\left\|\boldsymbol{\pi}_{h}^{n+1}-\boldsymbol{\pi}_{h}^{n}\right\|^{2}\right), \\
\left(\nabla p_{h}^{n+1}-\pi_{h}^{n+\beta}, \nabla \delta p_{h}^{n}\right) & =\left(\boldsymbol{\pi}_{3}^{n+1}, \nabla \delta p_{h}^{n}\right)+\bar{\beta}\left(\boldsymbol{\pi}_{h}^{n+1}-\boldsymbol{\pi}_{h}^{n}, \nabla \delta p_{h}^{n}\right) \\
& =\frac{1}{2}\left(\left\|\boldsymbol{\pi}_{3}^{n+1}\right\|^{2}-\left\|\boldsymbol{\pi}_{3}^{n}\right\|^{2}+\left\|\boldsymbol{\pi}_{3}^{n+1}-\boldsymbol{\pi}_{3}^{n}\right\|^{2}\right)+\bar{\beta}\left\|\boldsymbol{\pi}_{h}^{n+1}-\boldsymbol{\pi}_{h}^{n}\right\|^{2} .
\end{aligned}
$$

Using these expressions in (55)-(57), the result in (54), and summing for $n$ it is found that

$$
\begin{gathered}
\left\{\boldsymbol{u}_{h}^{n}\right\} \in \ell^{\infty}\left(L^{2}\right), \quad\left\{\hat{\boldsymbol{u}}_{h}^{n+1 / 2}\right\} \in \ell^{2}\left(H^{1}\right), \\
\left\{\delta t \nabla p_{h}^{n}\right\} \in \ell^{\infty}\left(L^{2}\right), \quad\left\{\sqrt{\delta t} \nabla \delta p_{h}^{n}\right\} \in \ell^{2}\left(L^{2}\right), \quad\left\{\sqrt{\tau} \pi_{3}^{n}\right\} \in \ell^{2}\left(L^{2}\right) .
\end{gathered}
$$

On the other hand, from (53), the inequality $a b \leq\left(a^{2}+b^{2}\right) / 2$ and these stability results it is readily seen that

$$
\left\|\hat{\boldsymbol{u}}_{h}^{n+1}\right\|^{2} \leq \frac{1}{2}\left\|\hat{\boldsymbol{u}}_{h}^{n+1}\right\|^{2}+C\left(\left\|\boldsymbol{u}_{h}^{n}\right\|^{2}+\delta t^{2}\left\|\nabla p_{h}^{n}\right\|^{2}+\delta t\left\|\boldsymbol{f}^{n+1 / 2}\right\|_{-1}^{2}\right),
$$

from where $\left\{\hat{\boldsymbol{u}}_{h}^{n}\right\} \in \ell^{\infty}\left(L^{2}\right)$.

It remains to bound $\pi_{1}^{n}$ in $\ell^{1}\left(L^{2}\right)$. Using the same strategy as for the first-order scheme (see (49)), we now have that

$$
\begin{aligned}
\delta t & \sqrt{\tau}\left\|\boldsymbol{\pi}_{1}^{n+1}\right\| \leq \sqrt{\tau}\left\|\boldsymbol{u}_{h}^{n+1}-\boldsymbol{u}_{h}^{n}\right\|+\delta t\left(N_{a}\left\|\hat{\boldsymbol{u}}_{h}^{n+1 / 2}\right\|_{1}+N_{c}\left\|\hat{\boldsymbol{u}}_{h}^{n+1 / 2}\right\|_{1}^{2}\right) \\
& +\delta t\left\|\boldsymbol{f}^{n+1 / 2}\right\|_{-1} .
\end{aligned}
$$

When summing for $n$, the only term that still has not been shown to be bounded is the first on the right-hand side. Bounding it will conclude the proof. Since

$$
\sum_{n=0}^{N-1} \sqrt{\tau}\left\|\boldsymbol{u}_{h}^{n+1}-\boldsymbol{u}_{h}^{n}\right\| \leq \sum_{n=0}^{N-1} \sqrt{\tau}\left\|\boldsymbol{u}_{h}^{n+1}-\hat{\boldsymbol{u}}_{h}^{n+1}\right\|+\sum_{n=0}^{N-1} \sqrt{\tau}\left\|\hat{\boldsymbol{u}}_{h}^{n+1}-\boldsymbol{u}_{h}^{n}\right\|
$$

and, from (40) and the assumption $\tau \leq C \delta t$,

$$
\begin{aligned}
\sum_{n=0}^{N-1} \sqrt{\tau}\left\|\boldsymbol{u}_{h}^{n+1}-\hat{\boldsymbol{u}}_{h}^{n+1}\right\| & =\sum_{n=0}^{N-1} \sqrt{\tau} \delta t\left\|\boldsymbol{\pi}_{h}^{n+1}-\boldsymbol{\pi}_{1}^{n}\right\| \\
& \leq C \sum_{n=0}^{N-1} \delta t^{2}\left\|\nabla p_{h}^{n+1}-\nabla p_{h}^{n}\right\|^{2}<\infty,
\end{aligned}
$$

it only remains to bound the last term in (58). Taking $\boldsymbol{v}_{h}=\hat{\boldsymbol{u}}_{h}^{n+1}-\boldsymbol{u}_{h}^{n}$ in (50) and using an inverse estimate similar to (48), it is found that

$$
\begin{aligned}
\frac{1}{\delta t}\left\|\hat{\boldsymbol{u}}_{h}^{n+1}-\boldsymbol{u}_{h}^{n}\right\|^{2} & \leq \frac{1}{\sqrt{\tau}} A_{n}\left\|\hat{\boldsymbol{u}}_{h}^{n+1}-\boldsymbol{u}_{h}^{n}\right\|+B_{n}, \\
A_{n} & :=N_{a}\left\|\hat{\boldsymbol{u}}_{h}^{n+1 / 2}\right\|_{1}+N_{c}\left\|\hat{\boldsymbol{u}}_{h}^{n+1 / 2}\right\|_{1}^{2}+\left\|\boldsymbol{f}^{n+1 / 2}\right\|_{-1}, \\
B_{n} & :=\left(p_{h}^{n}, \nabla \cdot \hat{\boldsymbol{u}}_{h}^{n+1}\right)-\left(p_{h}^{n}, \nabla \cdot \boldsymbol{u}_{h}^{n}\right) .
\end{aligned}
$$


Using the fact that if $x^{2} \leq b x+c$ then $x \leq 2 b+2 \sqrt{c}$, we obtain

$$
\sum_{n=0}^{N-1} \sqrt{\tau}\left\|\hat{\boldsymbol{u}}_{h}^{n+1}-\boldsymbol{u}_{h}^{n}\right\| \leq C\left(\sum_{n=0}^{N-1} \delta t A_{n}+\sum_{n=0}^{N-1} \sqrt{\delta t} \sqrt{\tau} \sqrt{B_{n}}\right) .
$$

The first term on the right-hand side is bounded, and therefore it only remains to obtain a bound for the second. Since $\tau \leq C \delta t$,

$$
\sum_{n=0}^{N-1} \sqrt{\delta t} \sqrt{\tau} \sqrt{B_{n}} \leq C \sum_{n=0}^{N-1} \delta t B_{n}
$$

From (55) we have

$$
\begin{gathered}
-\sum_{n=0}^{N-1} \delta t\left(p_{h}^{n}, \nabla \cdot \boldsymbol{u}_{h}^{n}\right) \leq \frac{1}{2} \sum_{n=0}^{N-1} \delta t^{2}\left(\left\|\boldsymbol{\pi}_{23}^{n}\right\|^{2}-\left\|\boldsymbol{\pi}_{23}^{n-1}\right\|^{2}+\left\|\boldsymbol{\pi}_{23}^{n}-\boldsymbol{\pi}_{23}^{n-1}\right\|^{2}\right) \\
\quad+\sum_{n=0}^{N-1} \delta t \tau\left[\left\|\boldsymbol{\pi}_{3}^{n}\right\|^{2}+\frac{\bar{\beta}}{2}\left(\left\|\boldsymbol{\pi}_{h}^{n}\right\|^{2}-\left\|\boldsymbol{\pi}_{h}^{n-1}\right\|^{2}+\left\|\boldsymbol{\pi}_{h}^{n}-\boldsymbol{\pi}_{h}^{n-1}\right\|^{2}\right)\right]<\infty,
\end{gathered}
$$

and similarly to (55)-(57) we can obtain

$$
\begin{aligned}
\sum_{n=0}^{N-1} & \delta t\left(p_{h}^{n}, \nabla \cdot \hat{\boldsymbol{u}}_{h}^{n+1}\right) \\
= & \sum_{n=0}^{N-1}\left[-\delta t^{2}\left(\nabla \delta p_{h}^{n}, \nabla p_{h}^{n}\right)-\delta t \tau\left(\nabla p_{h}^{n+1}-\boldsymbol{\pi}_{h}^{n+\beta}, \nabla p_{h}^{n}\right)\right] \\
= & \sum_{n=0}^{N-1}\left[\frac{\delta t^{2}}{2}\left(\left\|\nabla p_{h}^{n}\right\|^{2}-\left\|\nabla p_{h}^{n+1}\right\|^{2}+\left\|\nabla p_{h}^{n}-\nabla p_{h}^{n+1}\right\|^{2}\right)\right. \\
& \left.-\delta t \tau\left(\pi_{3}^{n+1}, \boldsymbol{\pi}_{3}^{n}\right)-\delta t \tau \bar{\beta}\left(\boldsymbol{\pi}_{h}^{n+1}-\boldsymbol{\pi}_{h}^{n}, \boldsymbol{\pi}_{h}^{n}\right)\right] \\
\leq & C \sum_{n=0}^{N-1} \delta t^{2}\left(\left\|\nabla p_{h}^{n}-\nabla p_{h}^{n+1}\right\|^{2}+\left\|\pi_{3}^{n+1}\right\|^{2}+\left\|\pi_{3}^{n}\right\|^{2}+\bar{\beta}\left\|\pi_{h}^{n+1}-\pi_{h}^{n}\right\|^{2}\right) .
\end{aligned}
$$

From the results already obtained, the last term on the right-hand side is bounded. This completes the proof of stability.

\section{PRESSURE AND CONVECTION STABILIZATION}

The pressure stabilization procedure introduced in the previous section consists of adding the term

$$
\tau\left(\nabla p_{h}^{n+1}-\pi_{h}^{n}, \nabla q_{h}\right)
$$

to the variational equations of the Galerkin method (either to the monolithic or to the fractional step time discretization). We have considered directly the case $\beta=0$, which is 
more appealing from the computational point of view and has the same stability properties as the case $\beta=1$. This stabilizing term can be thought of as a least-squares form of the component of the pressure gradient orthogonal to the finite element space, $\pi_{3}^{n+1}=$ $\nabla p_{h}^{n+1}-\pi_{h}^{n+1}$. If this allows the stabilization of the pressure, the idea that naturally arises is to use the same strategy to stabilize convection when the convective term dominates the viscous one. This idea is developed in [25]. Here we describe only the final outcome, which consists of adding

$$
\tau\left(\hat{\boldsymbol{u}}_{h}^{n+\theta} \cdot \nabla \hat{\boldsymbol{u}}_{h}^{n+\theta}+\nabla p_{h}^{n+1}-\boldsymbol{\pi}_{h}^{n}, \hat{\boldsymbol{u}}_{h}^{n+\theta} \cdot \nabla \boldsymbol{v}_{h}+\nabla q_{h}\right)
$$

to the variational equations of the Galerkin method. The contribution to the momentum equation is obtained when the pressure test function is $q_{h}=0$, whereas the contribution to the continuity equation is found when $\boldsymbol{v}_{h}=\mathbf{0}$. The final result is that the equations to be solved when fractional step methods are used are

$$
\begin{gathered}
\frac{1}{\delta t}\left(\hat{\boldsymbol{u}}_{h}^{n+1}-\boldsymbol{u}_{h}^{n}, \boldsymbol{v}_{h}\right)+c\left(\hat{\boldsymbol{u}}_{h}^{n+\theta}, \hat{\boldsymbol{u}}_{h}^{n+\theta}, \boldsymbol{v}_{h}\right)+a\left(\hat{\boldsymbol{u}}_{h}^{n+\theta}, \boldsymbol{v}_{h}\right)-\gamma b\left(p_{h}^{n}, \boldsymbol{v}_{h}\right) \\
+\tau\left(\hat{\boldsymbol{u}}_{h}^{n+\theta} \cdot \nabla \hat{\boldsymbol{u}}_{h}^{n+\theta}+\nabla p_{h}^{n}-\boldsymbol{\pi}_{h}^{n}, \hat{\boldsymbol{u}}_{h}^{n+\theta} \cdot \nabla \boldsymbol{v}_{h}\right)=\left\langle\boldsymbol{f}^{n+\theta}, \boldsymbol{v}_{h}\right\rangle \quad \forall \boldsymbol{v}_{h} \in \boldsymbol{V}_{h}, \\
-\delta t\left(\nabla\left(p_{h}^{n+1}-\gamma p_{h}^{n}\right), \nabla q_{h}\right)-\tau\left(\hat{\boldsymbol{u}}_{h}^{n+\theta} \cdot \nabla \hat{\boldsymbol{u}}_{h}^{n+\theta}+\nabla p_{h}^{n+1}-\boldsymbol{\pi}_{h}^{n}, \nabla q_{h}\right) \\
=\left(q_{h}, \nabla \cdot \hat{\boldsymbol{u}}_{h}^{n+1}\right) \quad \forall q_{h} \in Q_{h}, \\
\frac{1}{\delta t}\left(\boldsymbol{u}_{h}^{n+1}-\hat{\boldsymbol{u}}_{h}^{n+1}, \boldsymbol{v}_{h}\right)-b\left(p_{h}^{n+1}-\gamma p_{h}^{n}, \boldsymbol{v}_{h}\right)=0 \quad \forall \boldsymbol{v}_{h} \in \boldsymbol{V}_{h}, \\
\quad\left(\boldsymbol{\pi}_{h}^{n+1}, \boldsymbol{\eta}_{h}\right)=\left(\hat{\boldsymbol{u}}_{h}^{n+\theta} \cdot \nabla \hat{\boldsymbol{u}}_{h}^{n+\theta}+\nabla p_{h}^{n+1}, \boldsymbol{\eta}_{h}\right) \quad \forall \boldsymbol{\eta}_{h} \in \tilde{\boldsymbol{V}}_{h},
\end{gathered}
$$

which replace (36)-(39). It has to be observed that the pressure in the first equation is treated explicitly, in order to keep the uncoupling of the velocity and pressure calculations. Observe also that now $\boldsymbol{\pi}_{h}$ has the meaning of being the projection of $\hat{\boldsymbol{u}}_{h} \cdot \nabla \hat{\boldsymbol{u}}_{h}+\nabla p_{h}$ onto the finite element space.

Let us finally remark that the possibility of treating explicitly the projection $\pi_{h}$ greatly simplifies the numerical implementation of this stabilization technique. Apart from the evaluation of the several additional terms in the equations for the velocity and the pressure, only an $L^{2}$ projection needs to be performed at the end of each time step. This is computationally very inexpensive, especially if the Gramm matrix $\tilde{M}$ involved in the process is approximated by the diagonal one obtained from a standard lumping technique.

\section{NUMERICAL RESULTS}

In this section we present the result of two simple 2-D numerical experiments to illustrate the practical impact of the theoretical findings. The objective of the first is to show the dependence of the pressure stability on the time step, whereas the second is mainly intended to check the accuracy, a point that has been mentioned but not analyzed.

\subsection{Cavity Flow Problem}

This example is the classical cavity flow problem. The Navier-Stokes equations are solved in the unit square with zero velocities everywhere on the boundary except on the 
top, where a tangent unit velocity is prescribed. The viscosity has been taken as $v=0.01$, which yields a Reynolds number of 100 . A mesh of $20 \times 20$ four-noded bilinear elements has been employed. The flow equations have been advanced in time until the steady-state has been reached. The critical time step for the explicit scheme has been estimated to be

$$
\delta t_{\text {crit }}=\left(\frac{4 v}{h^{2}}+\frac{2 U}{h}\right)^{-1}
$$
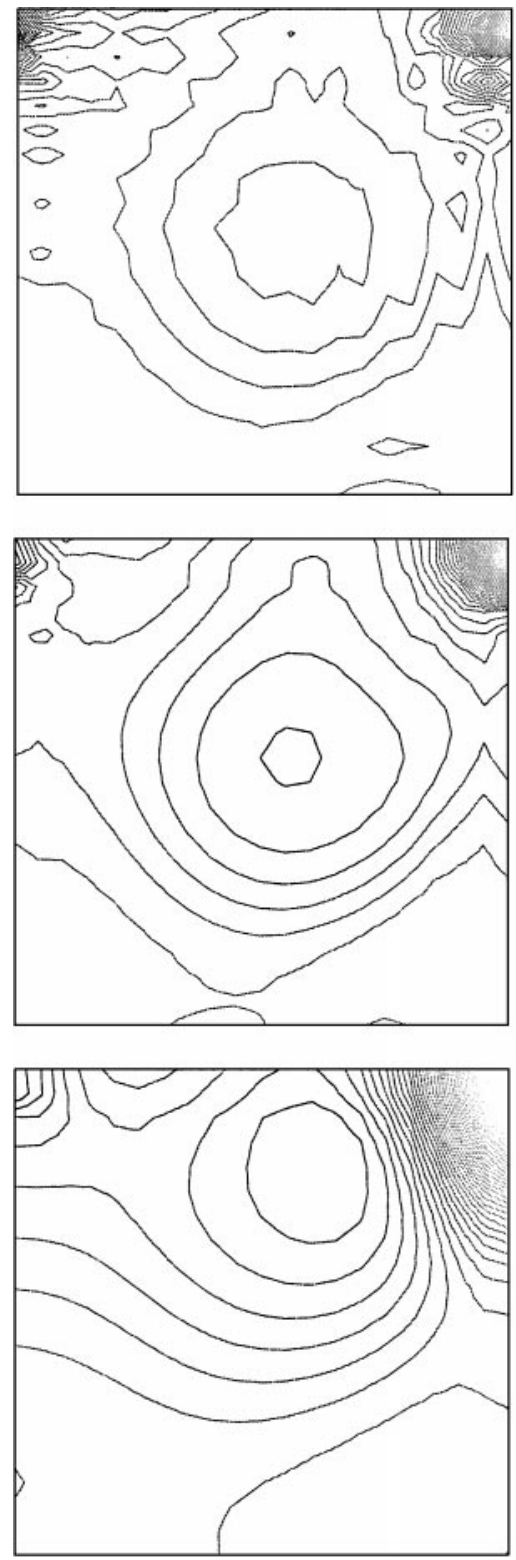

FIG. 1. Pressure contours for the cavity flow-problem using the first-order scheme. From the top to bottom: $\delta t=0.1 \delta t_{\text {crit }}, \delta t=\delta t_{\text {crit }}$, and $\delta t=1.0$. 

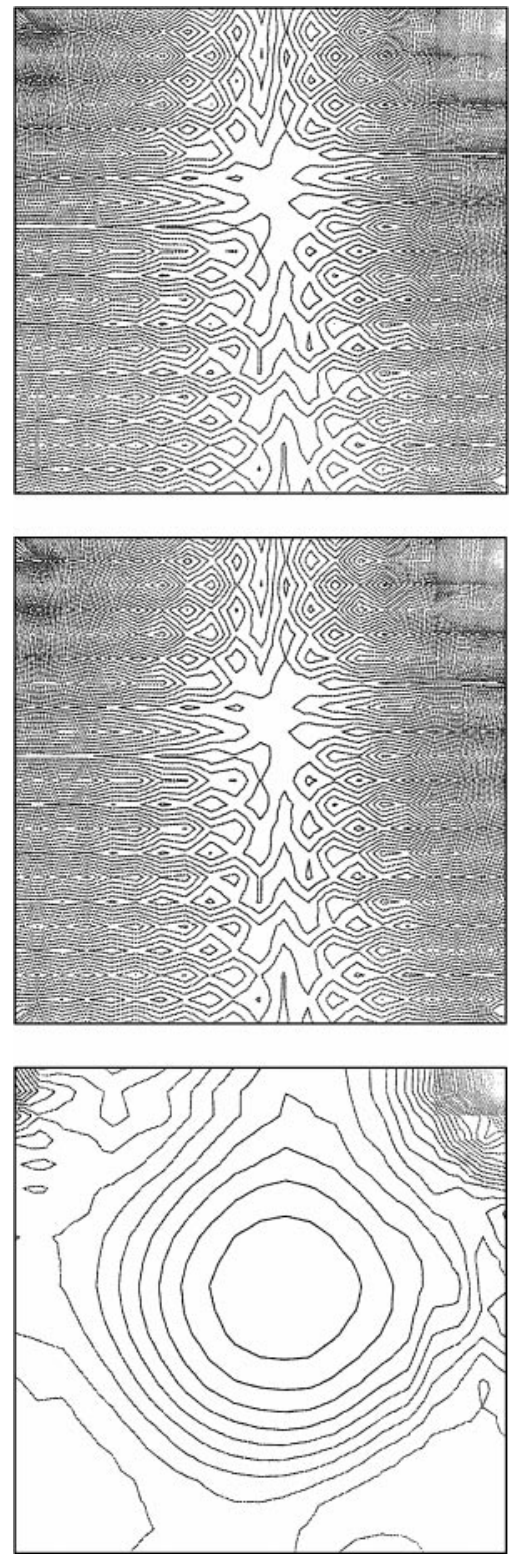

FIG. 2. Pressure contours for the cavity flow problem using the second-order scheme. From the top the bottom: $\delta t=0.1 \delta t_{\text {crit }}, \delta t=\delta t_{\text {crit }}$, and $\delta t=1.0$.

where $h=0.05$ is the element size and $U=1$ the characteristic velocity. This yields $\delta t_{\text {crit }}=1 / 56$.

Pressure contours using the original first-order scheme are shown in Fig. 1. It is clearly observed there that for $\delta t=0.1 \delta t_{\text {crit }}$ some oscillations appear, whereas for $\delta t=\delta t_{\text {crit }}$ the solution is acceptable. Likewise, when $\delta t$ is large ( 1 in this case), the solution is definitely overdiffusive. This shows the dependence of the pressure stability on the time step size.

The same cases have been computed with the second-order scheme. From Fig. 2, it is seen that only for very large values of $\delta t$ is the weak stability inherent to the scheme activated. 

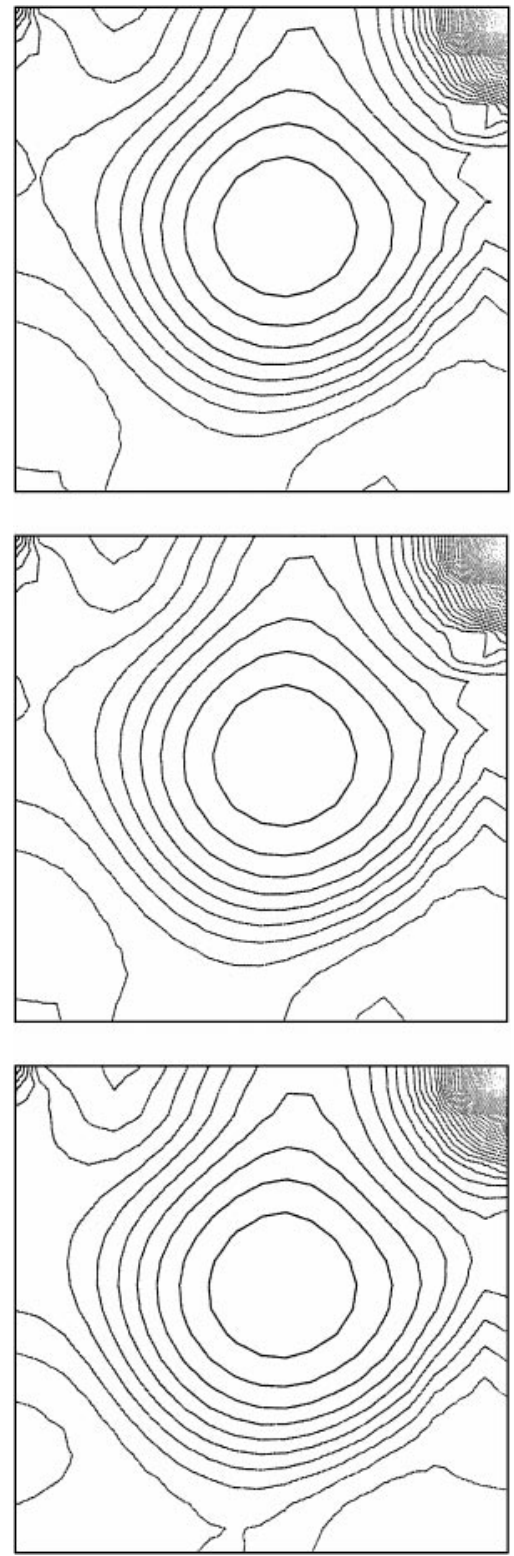

FIG. 3. Pressure contours for the cavity flow problem using the second-order scheme with stabilization. From the top to the bottom: $\delta t=0.1 \delta t_{\text {crit }}, \delta t=\delta t_{\text {crit }}$, and $\delta t=1.0$.

Contrary to the previous case, for $\delta t=0.1 \delta t_{\text {crit }}$ and $\delta t=\delta t_{\text {crit }}$ the pressure solution is completely oscillatory.

The final set of results for this problem shown in Fig. 3 corresponds to the stabilized formulation using the second-order scheme. The stability parameter $\tau$ has been computed as the critical time step given by (59) but for each element, taking $U$ as the mean element velocity. The pressure solution is correct for all the values of $\delta t$.

Even though the implicit schemes we have considered allow us to use time steps as large as desired, it is known that in practice the steady-state is reached faster (both in real time 

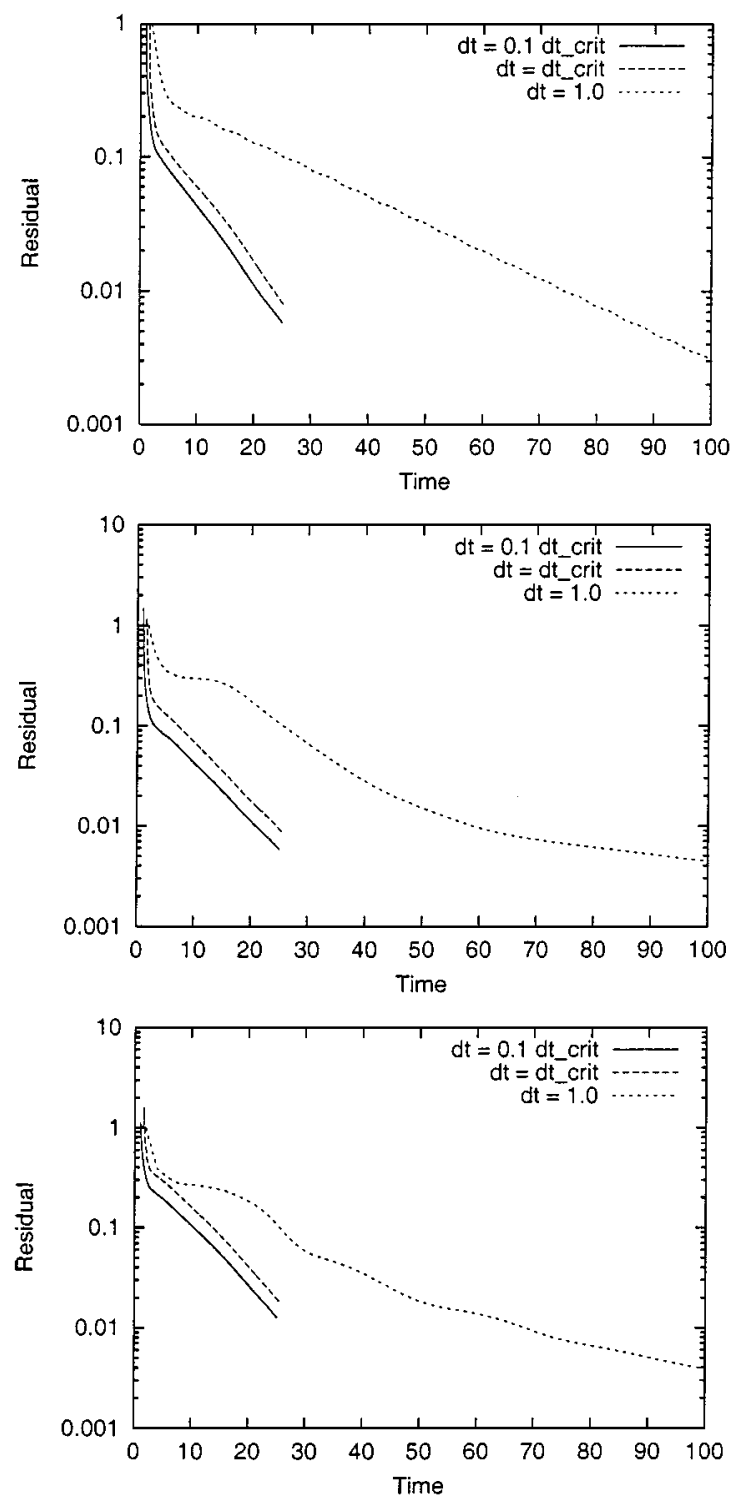

FIG. 4. Velocity convergence toward the steady-state for the cavity flow problem. From the top to the bottom: first-order scheme, second-order scheme, and second-order scheme with stabilization.

and in CPU time) using $\delta t$ close to $\delta t_{\text {crit }}$. We have also used this example to demonstrate this. Converge to the steady-state for the different formulations employed is shown in Fig. 4, where the residual is measured as $\left|U^{n}-U^{n-1}\right| /\left|U^{1}-U^{0}\right|$ (here $|\cdot|$ is the standard Euclidean norm of an array). From Fig. 5 it is seen that this dependence on $\delta t$ is similar for all the schemes.

\subsection{A Test with Analytical Solution}

We have referred to the two formulations analyzed throughout the paper as the first-order projection method and a second-order scheme. In this example we test the accuracy of 

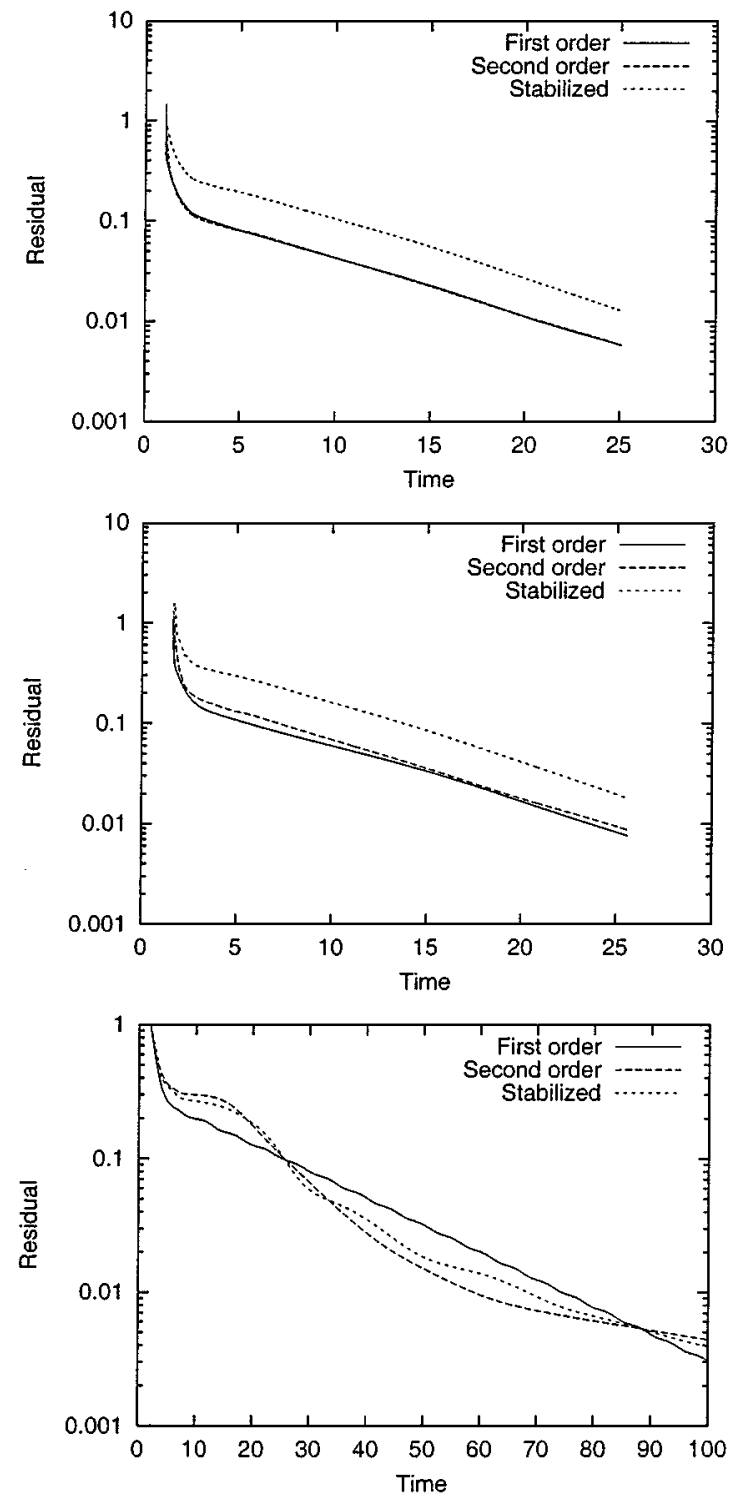

FIG. 5. Velocity convergence toward the steady-state for the cavity flow problem. From the top to the bottom: $\delta t=0.1 \delta t_{\text {crit }}, \delta t=\delta t_{\text {crit }}$, and $\delta t=1.0$.

these formulations. Since we have seen that the second-order one is unstable, we have combined it with the pressure stabilization technique, with the parameter $\tau$ computed as in the previous test.

In this example we solve again the Navier-Stokes equations in the unit square with homogeneous velocity conditions and taking the force vector so as to have as exact solution

$$
\begin{gathered}
u_{1}\left(x_{1}, x_{2}, t\right)=f\left(x_{1}\right) f^{\prime}\left(x_{2}\right) g(t), \quad u_{1}\left(x_{1}, x_{2}, t\right)=-f^{\prime}\left(x_{1}\right) f\left(x_{2}\right) g(t), \\
f(x)=100 x^{2}(1-x)^{2}, \quad g(t)=\cos (\pi t) \exp (-t),
\end{gathered}
$$




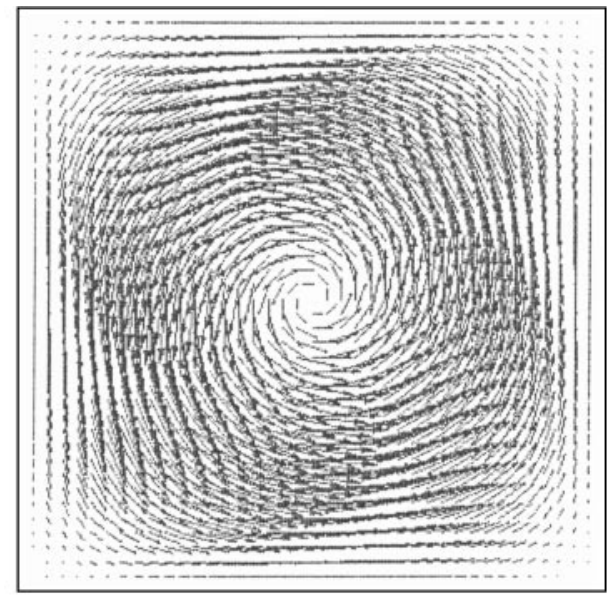

FIG. 6. Velocity vectors for the test with analytical solution.

with two solutions for the pressure, namely, $p=0$ and $p=100 x^{2}$. The time interval of analysis is $[0,1]$ and the viscosity $v=0.1$. A mesh of $40 \times 40$ bilinear elements has been employed to discretize the computational domain. The velocity solution at $t=1$ is shown in Fig. 6.

Convergence of the time approximation for the case $p=0$ and measured at $t=1$ is plotted in Fig. 7, where "total" refers to the scheme with $\gamma=0$ in (15)-(17), and "incremental" refers to the case $\gamma=1$. The error has been computed as $\left|U_{t=1}-U_{\text {exact }}\right| /\left|\bigcup_{\text {exact }}\right|$, where $\bigcup_{t=1}$ is the numerical solution at $t=1$, and $\bigcup_{\text {exact }}$ is the array of nodal values of the exact velocity at $t=1$. Since the exact pressure is $p=0$, the value of $\gamma$ does not affect the

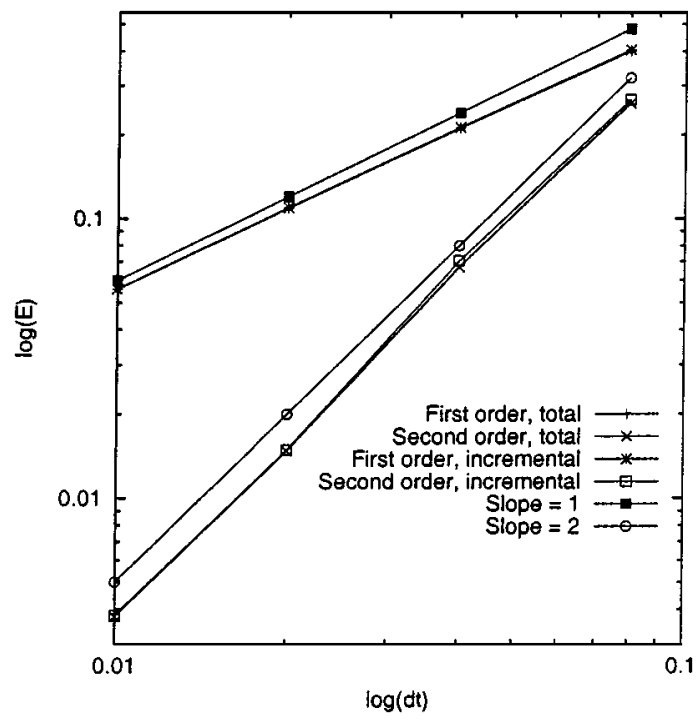

FIG. 7. Convergence for the test with analytical solution. Case $p=0$. 


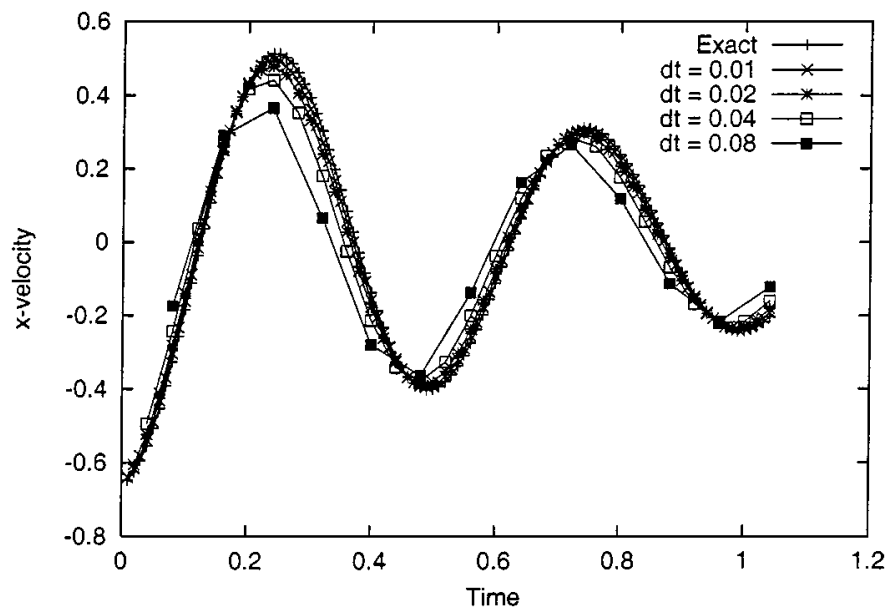

FIG. 8. Evolution of the $x$-velocity at $(0.75,0.75)$ using the first-order scheme. Case $p=0$.

accuracy in this example. From Fig. 7 it is seen that $\theta=1$ gives a first-order approximation and $\theta=1 / 2$ a second-order one. The evolution of the first velocity component $u_{1}$ at a point is depicted in Fig. 8 and Fig. 9 for the first- and second-order schemes, respectively.

The evolution to the steady-state when $g(t)$ is replaced by 1 in (60) is shown in Fig. 10. As in the previous example, it is seen that large values of $\delta t$ may yield slower rates of convergence. Finally, Fig. 11 shows the error at the steady-state as a function of $\delta t$ when $p=100 x^{2}$, measuring the well-known property that for the first-order scheme the solution depends largely on $\delta t$.

\section{SUMMARY OF MAIN RESULTS AND CONCLUSIONS}

The main objective of this paper has been to clarify the role of the pressure Poisson equation in the pressure stability of fractional step methods for incompressible flows, in

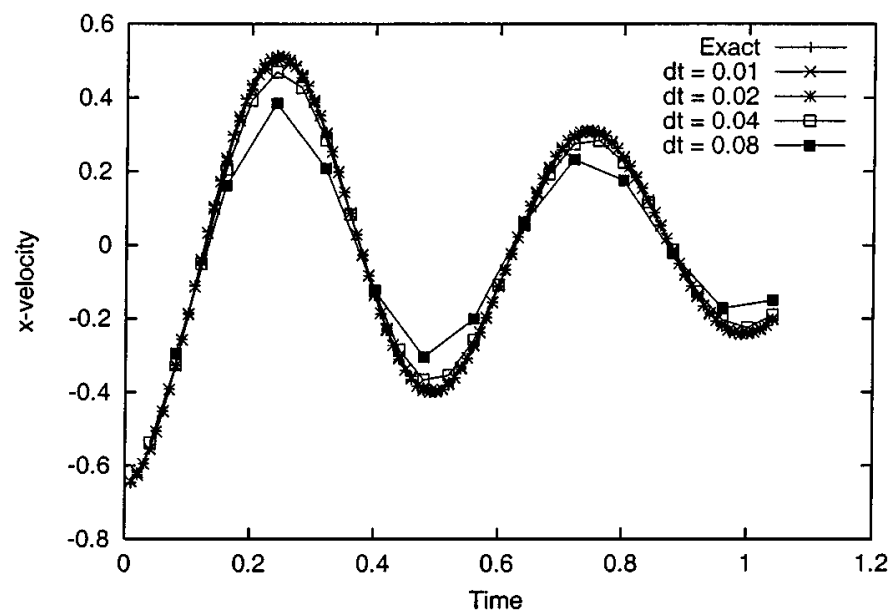

FIG. 9. Evolution of the $x$-velocity at $(0.75,0.75)$ using the second-order scheme. Case $p=0$. 


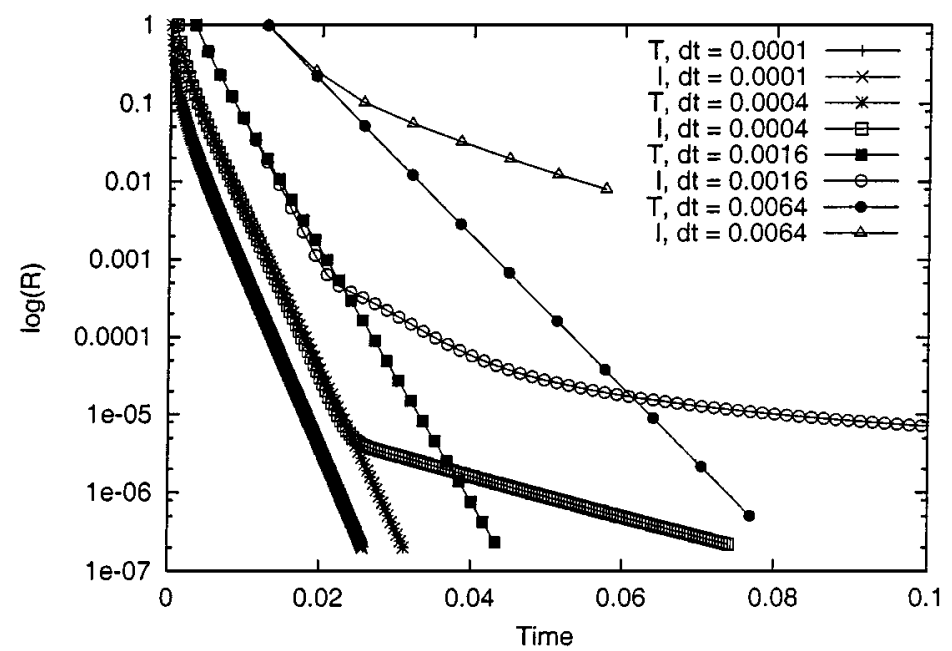

FIG. 10. Convergence to the steady-state for the test with analytical solution (T: total, I: incremental). Case $p=100 x^{2}$.

our case using the finite element method for the space discretization. The basic stability results for the two schemes analyzed are:

\begin{tabular}{c} 
First order: $\left\{\sqrt{\delta t} \nabla p_{h}^{n}\right\} \in \ell^{2}\left(L^{2}\right), \quad\left\{\sqrt{\tau} \nabla p_{h}^{n}\right\} \in \ell^{1}\left(L^{2}\right)$ \\
Second order: $\left\{\delta t \nabla p_{h}^{n}\right\} \in \ell^{\infty}\left(L^{2}\right), \quad\left\{\sqrt{\delta t} \nabla \delta p_{h}^{n}\right\} \in \ell^{2}\left(L^{2}\right), \quad\left\{\sqrt{\tau} \nabla p_{h}^{n}\right\} \in \ell^{1}\left(L^{2}\right)$ \\
\hline
\end{tabular}

Inspecting these results, the main conclusions that can be drawn include:

- For the original ( $\tau=0$ ) first-order scheme, pressure is stable, but the parameter that controls the amount of stability is the time step size, and therefore:

- If $\delta t$ is very small, pressure oscillations may appear.

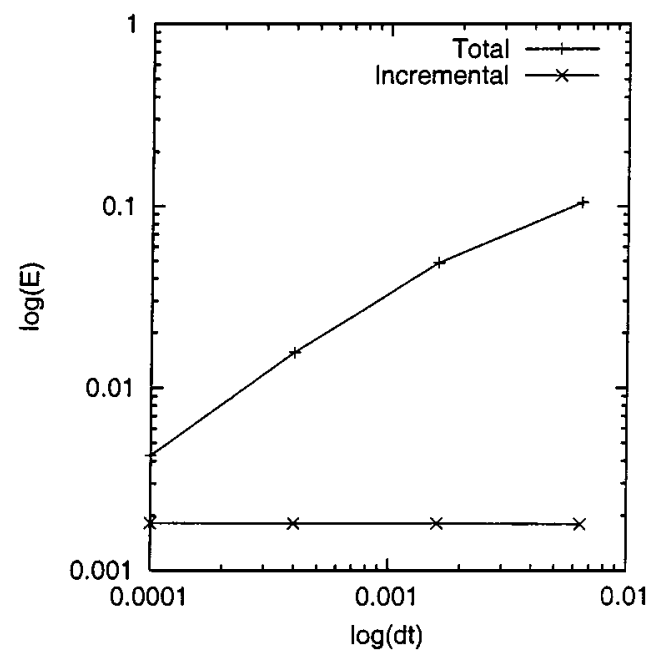

FIG. 11. Error at the steady-state as a function of the time step for the test with analytical solution. Case $p=100 x^{2}$. 
- If $\delta t$ is large, the method may be overdiffusive. This limits the applicability of implicit schemes, since appropriate values of $\delta t$ adequate for stability turn out to be close to the critical time step of explicit schemes $(\theta=0)$.

- The original ( $\tau=0$ ) second-order method has a very poor pressure stability. At the steady-state, only that $\delta t\left\|\nabla p_{h}\right\| \leq C<\infty$ can be ensured, whereas the optimum would be to have control on $\sqrt{\delta t}\left\|\nabla p_{h}\right\|$. However, if $\delta t$ is very large, the control on $\delta t\left\|\nabla p_{h}\right\|$ can be enough to obtain stable pressures.

- Pressure stability in stabilized schemes depends on an algorithmic parameter $\tau$, which may be chosen independent of $\delta t$ (except for the condition $\tau \leq C \delta t$ needed for the secondorder scheme). Both for the first- and for the second-order methods, this stabilization allows us to free the link stability- $\delta t$. For the first-order scheme, $\delta t$ can be arbitrarily small, and for the second-order one we improve the estimate $\left\{\delta t \nabla p_{h}^{n}\right\} \in \ell^{\infty}\left(L^{2}\right)$. In both cases, we are left with $\left\{\sqrt{\tau} \nabla p_{h}^{n}\right\} \in \ell^{1}\left(L^{2}\right)$.

A very important fact from the computational point of view is that the pressure gradient projection for stabilized schemes may be treated explicitly (which corresponds to taking $\beta=0$ in (37)). It has been shown that this does not upset stability. Finally, let us mention that convection dominated flows can be stabilized by considering a natural extension of the pressure stabilization technique which has been described in Section 5.

\section{REFERENCES}

1. A. J. Chorin, A numerical method for solving incompressible viscous problems, J. Comput. Phys. 2, 12 (1967).

2. R. Temam, Sur l'approximation de la solution des équations de Navier-Stokes par la méthode des pas fractionaires (I), Arch. Ration. Mech. Anal. 32, 135 (1969).

3. P. M. Gresho and R. L. Sani, Incompressible flow and the finite element method, (Wiley, New York, 2000).

4. R. Natarajan, A numerical method for incompressible viscous flow simulation, J. Comput. Phys. 100, 384 (1992).

5. J. Shen, Hopf bifurcation of the unsteady regularized driven cavity-flow, J. Comput. Phys. 95, 228 (1991).

6. S. Turek, A comparative study of time-stepping techniques for the incompressible Navier-Stokes equations: from fully implicit nonlinear schemes to semi-implicit projection methods. Int. J. Numer. Meth. Fluids 22, 987 (1996).

7. R. Temam, Remark on the pressure boundary condition for the projection method, Theor. Comput. Fluid Dyn. 3, 181 (1991).

8. P. M. Gresho, On the theory of semi-implicit projection methods for viscous incompressible flow and its implementation via a finite element method that also introduces a nearly consistent mass matrix. I: Theory, Int. J. Numer. Meth. Fluids 11, 587 (1990).

9. J. B. Perot, An analysis of the fractional step method, J. Comput. Phys. 108, 51 (1993).

10. A. Quarteroni, F. Saleri, and A. Veneziani, Factorization methods for the numerical approximation of NavierStokes equations, Comput. Meth. Appl. Mech. Eng. 188, 505 (2000).

11. J. Shen, On error estimates of projection methods for Navier-Stokes equations: First order squemes, SIAM J. Numer. Anal. 29, 57 (1992).

12. J. Shen, On error estimates for some higher order projection and penalty-projection methods for Navier-Stokes equations, Numerische Mathematik 62, 49 (1992).

13. J. L. Guermond and L. Quartapelle, On the approximation of the unsteady Navier-Stokes equations by finite element projection methods, Numerische Mathematik 80, 207 (1998).

14. J. C. Strikwerda and Y. S. Lee, The accuracy of the fractional step method, SIAM J. Numer. Anal. 37, 37 (1999).

15. J. L. Guermond and L. Quartapelle, On stability and convergence of projection methods based on pressure Poisson equation, Int. J. Numer. Meth. Fluids 26, 1039 (1998). 
16. J. B. Bell, P. Colella, and H. M. Glaz, A second-order projection method for the incompressible Navier-Stokes equations, J. Comput. Phys. 85, 257 (1989).

17. G. E. Karniadakis, M. Israeli, and S. E. Orzag, High order splitting methods for the incompressible NavierStokes equations, J. Comput. Phys. 59, 414 (1991).

18. J. Kim and P. Moin, Application of the fractional step method to incompressible Navier-Stokes equations, $J$. Comput. Phys. 59, 308 (1985).

19. J. van Kan, A second-order accurate pressure correction scheme for viscous incompressible flow, SIAM J. Sci. Stat. Comp. 7, 870 (1986).

20. P. M. Gresho, S. T. Chan, M. A. Christon and A. C. Hindmarsh, A little more on stabilized $Q_{1} Q_{1}$ for transient viscous incompressible flow, Int. J. Numer. Meth. Fluids 21, 837 (1995).

21. R. Codina, Numerical solution of the incompressible Navier-Stokes equations with Coriolis forces based on the discretization of the total time derivative, J. Comput. Phys. 148, 467 (1999).

22. R. Codina and J. Blasco, A finite element formulation for the Stokes problem allowing equal velocity-pressure interpolation, Comput. Meth. Appl. Mech. Eng. 143, 373 (1997).

23. R. Codina and J. Blasco, Analysis of a pressure-stabilized finite element approximation of the stationary Navier-Stokes equations, Numerische Mathematik 87, 59 (2000).

24. R. Codina and J. Blasco, Stabilized finite element method for the transient Navier-Stokes equations based on a pressure gradient projection, Comput. Meth. Appl. Mech. Eng. 182, 287 (2000).

25. R. Codina, Stabilization of incompressibility and convection through orthogonal sub-scales in finite element methods, Comp. Meth. Appl. Mech. Eng. 190, 1579 (2000).

26. J. G. Heywood and R. Rannacher, Finite element approximation of the nonstationary Navier-Stokes problem. IV: Error analysis for second-order time discretization, SIAM J. Numer. Anal. 27, 353 (1990).

27. F. Brezzi and M. Fortin, Mixed and Hybrid Finite Element Methods (Springer-Verlag, Berlin/New York, 1991).

28. G. E. Schneider, G. D. Raithby, and M. M. Yovanovich, Finite element analysis of incompressible fluid flow incorporating equal order pressure and velocity interpolation, in Numerical Methods in Laminar and Turbulent Flow, edited by C. Taylor, K. Morgan, C. A. Brebbia (Pentech Press, Plymouth, 1978).

29. M. Kawahara and K. Ohmiya, Finite element analysis of density flow using the velocity correction method, Int. J. Numer. Meth. Fluids 5, 981 (1985).

30. O. C. Zienkiewicz and R. Codina, A general algorithm for compressible and incompressible flow-Part I. The split, characteristic-based scheme, Int. J. Numer. Meth. Fluids, 20, 869 (1995).

31. H. G. Choi, H. Choi, and J. Y. Yoo, A fractional four-step finite element formulation of the unsteady incompressible Navier-Stokes equations using supg and linear equal-order element methods, Comput. Meth. Appl. Mech. Eng. 143, 333 (1997).

32. T. J. R. Hughes, L. P. Franca, and M. Balestra, A new finite element formulation for computational fluid dynamics: V. Circumventing the Babuška-Brezzi condition: a stable Petrov-Galerkin formulation for the Stokes problem accommodating equal-order interpolations, Comput. Meth. Appl. Mech. Eng. 59, 85 (1986).

33. L. Franca and R. Stenberg, Error analysis of some Galerkin least-squares methods for the elasticity equations, SIAM J. Numer. Anal. 28, 1680 (1991).

34. L. P. Franca and S. L. Frey, Stabilized finite element methods: II. The incompressible Navier-Stokes equations, Comput. Meth. Appl. Mech. Eng. 99, 209 (1992).

35. F. Brezzi and J. Douglas, Stabilized mixed methods for the Stokes problem, Numerische Mathematik 53, 225 (1988).

36. R. Rannacher, On Chorin's Projection Method for Incompressible Navier-Stokes Equations, Lecture Notes in Mathematics (Springer-Verlag, Berlin, 1992), Vol. 1530, pp. 167-183.

37. J. L. Guermond and L. Quartapelle, Calculation of incompressible flows by an unconditionally stable FEM algorithm, J. Comput. Phys. 132, 12 (1997).

38. R. Codina, M. Vázquez, and O. C. Zienkiewicz, A general algorithm for compressible and incompressible flow-Part III. The semi-implicit form, Int. J. Numer. Meth. Fluids 27, 13 (1998).

39. S. C. Brenner and L. R. Scott, The Mathematical Theory of Finite Element Methods (Springer-Verlag, Berlin/New York, 1994). 